\title{
VARIANCE OF THE NUMBER OF ZEROES OF SHIFT-INVARIANT GAUSSIAN ANALYTIC FUNCTIONS
}

\author{
NAOMI DVORA FELDHEIM
}

\begin{abstract}
Following Wiener, we consider the zeroes of Gaussian analytic functions in a strip in the complex plane, with translation-invariant distribution. We show that the variance of the number of zeroes in a long horizontal rectangle $[-T, T] \times[a, b]$ is asymptotically between $c T$ and $C T^{2}$, with positive constants $c$ and $C$. We also supply with conditions (in terms of the spectral measure) under which the variance asymptotically grows linearly with $T$, as a quadratic function of $T$, or has intermediate growth.
\end{abstract}

\section{INTRODUCTION}

Consider complex Gaussian Analytic Functions (GAFs) whose distribution is invaruiant under real shifts. Paley and Wiener dedicate the last chapter of their celebrated treatise [15, Ch. X] to study the asymptotic number of zeroes of such functions in a long horizontal rectangle $[-T, T] \times[a, b]$ as $T$ tends to infinity. They show that under certain spectral conditions this quantity satisfies a law of large numbers. This result was extended in [7], where unnecessary conditions were removed, and a formula for the expected number of zeroes was developed.

This paper is devoted to the study of the fluctuations in the number of zeroes in the same setting. We study the asymptotic growth of the variance $V(T)$ of the number of zeroes in $[-T, T] \times[a, b]$. In particular we identify which processes demonstrate quadratic growth of $V(T)$ (Theorem 1), and supply sufficient and necessary conditions for $V(T)$ to be asymptotically linear (Theorem 2 and 3 respectively). In Theorem 2 we also prove that $V(T)$ is always at least linear in $T$. Obtaining lower bounds on the variance is the most difficult part of this paper (as was the case in related models, see discussion below).

1.1. Discussion. Zeroes of random Gaussian functions in increasing domains have been studied in various settings. Generally analyizing fluctuations is much harder than calculating expectations and even than obtaining central limit theorems. For real Gaussian stationary processes, a formula for the expectation was obtained in the early works of Kac [11 and

2000 Mathematics Subject Classification. 30E99, 60G15, 60G10.

Key words and phrases. Gaussian Analytic function, Stationary Gaussian process, fluctuations of zeroes. Department of Mathematics, Stanford University. Email: naomifel@stanford.edu.

Research supported by the Science Foundation of the Israel Academy of Sciences and Humanities, grant 166/11; by the United States - Israel Binational Science Foundation, grant 2012037; and by a National Science Foundation postdoctoral fellowship grant. 
Rice [17] from the 1940's. Cramer and Leadbetter [4] later obtained an asymptotic formula for the variance, but the rate of growth could not be inferred from it. In 1976 Cuzick [5] was able to show that, under some technical assumptions, if the growth of the variance is linear then the number of zeroes in $[-T, T]$ satisfies a Central Limit Theorem (CLT). It was only fifteen years later that Slud [18] obtained accessible conditions (in terms of the covariance kernel) for this assumption to hold. To do so Slud used primarily a sophisticated method for stochastic integration which he developed jointly with Chambers [3]. Unfortunately, this method involves many computations specifically tailored to tackle the real case, which do not generalize to the complex setting easily. Consequently, while some of our results for the complex case are very similar to those of Slud (see Remark 1.2 below), our methods are quite different, and, in a sense, more elementary.

Similar methods to those of Cuzick and Slud were recently used by Granville and Wigman [9], to study the number of zeroes of a Gaussian trigonometric polynomial of large degree $N$ in the interval $[0,2 \pi]$. They showed that the variance of this number is linear in $N$ and that a CLT holds.

In the complex setting Sodin-Tsirelson [19] and Nazarov-Sodin [14] studied the unique planar GAF whose zeroes are invariant under all planar isometries. They showed linear growth of the variance and a CLT for the zeroes in large balls (as the radius approaches infinity), using diagram counting methods.

In contrast to these works, this paper requires but standard tools from harmonic analysis, classical analysis and probability. In order to acquire our results we derive an asymptotic formula for $V(T)$ (Proposition 2). This formula consists of a series of non-negative terms involving the spectral measure, and is therefore relatively easy to analyse. Ideas from this paper were already used for studying the winding number of Gaussian functions from $\mathbb{R}$ to $\mathbb{C}$, in our work with Buckley [2].

1.2. Definitions. A Gaussian Analytic Function $(G A F)$ in the strip $D=D_{\Delta}=\{z:|\operatorname{Im} z|<$ $\Delta\}$ is a random variable taking values in the space of analytic functions on $D$, so that for every $n \in \mathbb{N}$ and every $z_{1}, \ldots, z_{n} \in D$ the vector $\left(f\left(z_{1}\right), \ldots, f\left(z_{n}\right)\right)$ has a mean zero complex Gaussian distribution.

A GAF in $D$ is called stationary, if it is distribution-invariant with respect to all horizontal shifts, i.e., for any $t \in \mathbb{R}$, any $n \in \mathbb{N}$, and any $z_{1}, \ldots, z_{n} \in D$, the random $n$-tuples

$$
\left(f\left(z_{1}\right), \ldots, f\left(z_{n}\right)\right) \quad \text { and } \quad\left(f\left(z_{1}+t\right), \ldots, f\left(z_{n}+t\right)\right)
$$

have the same distribution.

For a stationary GAF, the covariance kernel

$$
K(z, w)=\mathbb{E}\{f(z) \overline{f(w)}\}
$$

may be written as

$$
K(z, w)=r(z-\bar{w}) .
$$


For $t \in \mathbb{R}$, the function $r(t)$ is positive-definite and continuous, and so it is the Fourier transform of some positive measure $\rho$ on the real line:

$$
r(t)=\mathcal{F}[\rho](t)=\int_{\mathbb{R}} e^{-2 \pi i t \lambda} d \rho(\lambda)
$$

Moreover, since $r(t)$ has an analytic continuation to the strip $D_{2 \Delta}, \rho$ must have a finite exponential moment:

$$
\text { for each }\left|\Delta_{1}\right|<\Delta, \quad \int_{-\infty}^{\infty} e^{2 \pi \cdot 2 \Delta_{1}|\lambda|} d \rho(\lambda)<\infty .
$$

The measure $\rho$ is called the spectral measure of $f$. A stationary GAF is degenerate if its spectral measure consists of exactly one atom.

For a holomorphic function $f$ in a domain $D$, we denote by $Z_{f}$ the zero-set of $f$ (counted with multiplicities), and by $n_{f}$ the zero-counting measure, i.e.,

$$
\forall \varphi \in C_{0}(D): \quad \int_{D} \varphi(z) d n_{f}(z)=\sum_{z \in Z_{f}} \varphi(z),
$$

where $C_{0}(D)$ is the set of compactly supported continuous functions on $D$. We use the abbreviation $n_{f}(B)=\int_{B} d n_{f}(z)$ for the number of zeroes in a Borel subset $B \subset D$.

1.3. Results. First, we present a previous result which will serve as our starting point. This result can be viewed as a "law of large numbers" for the zeroes of stationary functions.

Theorem A. [7, Theorem 1] Let $f$ be a stationary non-degenerate GAF in the strip $D_{\Delta}$, where $0<\Delta \leq \infty$. Let $\nu_{f, T}$ be the non-negative locally-finite random measure on $(-\Delta, \Delta)$ defined by

$$
\nu_{f, T}(Y)=\frac{1}{2 T} n_{f}([-T, T) \times Y), Y \subset(-\Delta, \Delta) \text { measurable. }
$$

Then:

(i) Almost surely, the measures $\nu_{f, T}$ converge weakly and on every interval to a measure $\nu_{f}$ when $T \rightarrow \infty$.

(ii) The measure $\nu_{f}$ is not random (i.e. $\operatorname{var} \nu_{f}=0$ ) if and only if the spectral measure $\rho_{f}$ has no atoms.

(iii) If the measure $\nu_{f}$ is not random, then $\nu_{f}(Y)=\mathbb{E} n_{f}([0,1] \times Y)$ and it has density:

$$
L(y)=\frac{d}{d y}\left(\frac{\int_{-\infty}^{\infty} \lambda e^{4 \pi y \lambda} d \rho(\lambda)}{\int_{-\infty}^{\infty} e^{4 \pi y \lambda} d \rho(\lambda)}\right)=\frac{1}{4 \pi} \frac{d^{2}}{d y^{2}} \log (r(2 i y)) .
$$

In the above and in what follows, the term "density" means the Radon-Nikodym derivative w.r.t. the Lebegue measure on $\mathbb{R}$.

A natural question is, how big are the fluctuations of the number of zeroes in a long rectangle? More rigorously, define

$$
R_{T}^{a, b}=[-T, T) \times[a, b], \quad V_{f}^{a, b}(T)=\operatorname{var}\left[n_{f}\left(R_{T}^{a, b}\right)\right],
$$


where for a random variable $X$ the variance is defined by

$$
\operatorname{var}(X)=\mathbb{E}(X-\mathbb{E} X)^{2} .
$$

We are interested in the asymptotic behavior of $V_{f}^{a, b}(T)$ as $T$ approaches infinity. The next theorems show that $V_{f}^{a, b}(T)$ is asymptotically bounded between $c T$ and $C T^{2}$ for some $c, C>0$, and give conditions under which each of the bounds is achieved. We begin by stating the upper bound result, a relatively easy consequence of Theorem A,

Theorem 1. Let $f$ be a non-degenerate stationary GAF in a strip $D_{\Delta}$. Then for all $-\Delta<$ $a<b<\Delta$ the limit

$$
L_{2}=L_{2}(a, b):=\lim _{T \rightarrow \infty} \frac{V_{f}^{a, b}(T)}{T^{2}} \in[0, \infty)
$$

exists. This limit is positive if and only if the spectral measure of $f$ has a non-zero discrete component.

The lower bound result, which is our main result, is stated in the following theorem.

Theorem 2. Let $f$ be a non-degenerate stationary GAF in a strip $D_{\Delta}$. Then for all $-\Delta<$ $a<b<\Delta$ the limit

$$
L_{1}=L_{1}(a, b):=\lim _{T \rightarrow \infty} \frac{V_{f}^{a, b}(T)}{T} \in(0, \infty]
$$

exists. Moreover, the limit $L_{1}(a, b)$ is finite if $\rho$ is absolutely continuous with density $d \rho(\lambda)=$ $p(\lambda) d \lambda$, such that

$$
\left(1+\lambda^{2}\right) e^{2 \pi \cdot 2 y \lambda} p(\lambda) \in L^{2}(\mathbb{R}), \text { for } y \in\{a, b\}
$$

Several remarks are due before continuing.

Remark 1.1. Another form of condition (2) is the following: For $y \in\{2 a, 2 b\}$,

$$
\int_{\mathbb{R}}|r(x+i y)|^{2} d x, \quad \int_{\mathbb{R}}\left|r^{\prime \prime}(x+i y)\right|^{2} d x<\infty .
$$

This implies also that $\int_{\mathbb{R}}\left|r^{\prime}(x+i y)\right|^{2} d x<\infty$. Moreover, since the set $\left\{c: e^{2 \pi \cdot c \lambda} p(\lambda) \in L^{2}(\mathbb{R})\right\}$ is convex, it implies the same condition for all $y \in[2 a, 2 b]$.

Remark 1.2. It is interesting to note that condition (2) is precisely the condition that Slud gave in [18] for linear variance in the case of real (non-analytic) stationary Gaussian processes (with $a=b=0$ ). Nonetheless, no direct implication between the results is known and the methods to obtain them are quite remote.

As for the first part of Theorem 2, we would expect an analogue to hold for real startionary Gaussian functions; that is, that under mild conditions, the variance of the number of zeroes in $[-T, T]$ is always at least linear in $T$. To the best of our knowledge, this is yet unknown. 
Remark 1.3. In case condition (2) holds, we shall give an expression for the limit $L_{1}$ as a convergent series of the form:

$$
\lim _{T \rightarrow \infty} \frac{V_{f}^{a, b}(T)}{2 T}=\sum_{k \geq 1} \int_{\mathbb{R}}\left(p^{* k}(\lambda)\right)^{2} w_{k}^{a, b}(\lambda) d \lambda .
$$

Here $p^{* k}$ denotes the $k$-fold convolution of $p$, and $w_{k}^{a, b}(\lambda)$ is a positive function which can be computed explicitly in terms of $a, b, k$ and $r(2 i a), r(2 i b)$ (and no other reliance on $p$ ).

The next theorem deals with conditions under which $L_{1}(a, b)$ is infinite, i.e. the variance is super-linear.

Theorem 3. Let $f$ be a non-degenerate stationary GAF in a strip $D_{\Delta}$.

(i) Suppose $J \subset(-\Delta, \Delta)$ is a closed interval such that for every $y \in J$, the function $\lambda \mapsto\left(1+\lambda^{2}\right) e^{2 \pi \cdot 2 y \lambda} p(\lambda)$ does not belong to $L^{2}(\mathbb{R})$. Then for every $\alpha \in J$ the set $\left\{\beta \in J: L_{1}(\alpha, \beta)<\infty\right\}$ is finite.

(ii) The limit $L_{1}(a, b)$ is infinite for particular $a, b$ if either $\rho$ does not have density, or, if it has density $p$ and for any two points $\lambda_{1}, \lambda_{2} \in \mathbb{R}$ there exists intervals $I_{1}, I_{2}$ such that $I_{j}$ contains $\lambda_{j}(j=1,2)$ and

$$
(1+\lambda) e^{2 \pi \cdot 2 y \lambda} p(\lambda) \notin L^{2}\left(\mathbb{R} \backslash\left(I_{1} \cup I_{2}\right)\right),
$$

for at least one of the values $y=a$ or $y=b$.

Remark 1.4. There is a gap between the conditions given for linear variance (in Theorem 21) and those for super-linear variance (in Theorem 3 ). For instance, the theorems do not decide about all the suitable pairs $(a, b)$ in case the spectral measure has density $\frac{1}{\sqrt{|\lambda|}} \mathbb{I}_{[-1,1]}(\lambda)$. On the other hand, we are ensured to have super-linear variance in case $\rho$ has a singular part. If $\rho$ has density $p \in L^{1}(\mathbb{R})$ which is bounded on any compact set, then $\left(1+\lambda^{2}\right) p(\lambda) \in L^{2}(\mathbb{R})$ implies asymptotically linear variance, and $(1+\lambda) p(\lambda) \notin L^{2}(\mathbb{R})$ implies asymptotically superlinear variance.

Remark 1.5. Minor changes to the developments in this paper may be made in order to prove analogous results regarding the increment of the argument of a stationary GAF $f$ along a horizontal line. Namely, let $V^{a, a}(T)$ denote the variance of the increment of the argument of $f$ along the line $[0, T] \times\{a\}$ (for some $-\Delta<a<\Delta$ ). Then:

- the limit $L_{2}(a)=\lim _{T \rightarrow \infty} \frac{V^{a, a}(T)}{T^{2}}$ exists, belongs to $[0, \infty)$, and is positive if and only if the spectral measure contains an atom.

- the limit $L_{1}(a)=\lim _{T \rightarrow \infty} \frac{V^{a, a}(T)}{T}$ exists, belongs to $(0, \infty]$, and is finite if $\rho$ has density $p(\lambda)$ such that $\left(1+\lambda^{2}\right) e^{2 \pi \cdot 2 a \lambda} p(\lambda) \in L^{2}(\mathbb{R})$. Moreover, $L_{1}(a)$ is infinite if for any $\lambda_{0} \in \mathbb{R}$ there is an interval $I$ containing $\lambda_{0}$ such that the measure $(1+\lambda) e^{2 \pi \cdot 2 a \lambda} d \rho(\lambda)$ restricted to $\mathbb{R} \backslash I$ is not in $L^{2}(\mathbb{R})$. 
In our recent work with Buckley [2] we extend these statements to hold for a differentiable (not necessarily analytic) Gaussian process from $\mathbb{R}$ to $\mathbb{C}$. Also notice that the first item is essentially proved in this paper (Claim 3 below).

The rest of the paper is organized as follows: Theorem 1 concerning quadratic growth of variance is proved in Section 2, and is mainly a consequence of Theorem A. In Section 3 we develop an asymptotic formula for $V_{f}^{a, b}(T) / T$ (Proposition 2 below), which will be used to prove Theorems 2 and 3 in Sections 4 and 5 respectively. Appendices $\mathrm{A}$, B and $\mathrm{C}$ include proofs of some technical lemmas.

1.4. Acknowledgements. I thank Mikhail Sodin for his advice and encouragement during all stages of this work. I am grateful to Jeremiah Buckley, who contributed most of the arguments in Appendix B. I also thank Boris Tsirelson for his interest and suggestions which stimulated the research, and Boris Hanin for a useful discussion. I am grateful to Alon Nishry and Igor Wigman for reading the original draft carefully and pointing out some errors.

\section{Theorem 1: Quadratic Variance}

Recall the notation $R_{T}=R_{T}^{a, b}=[-T, T) \times[a, b]$. From Theorem $\mathrm{A}$ we know that

$$
\lim _{T \rightarrow \infty} \frac{n_{f}\left(R_{T}\right)}{T}=Z,
$$

where $Z$ is some random variable and the limit is in the almost sure sense. Moreover, $\operatorname{var} Z>0$ if and only if the spectral measure of $f$ contains an atom. Clearly

$$
\operatorname{var}\left(\lim _{T \rightarrow \infty} \frac{n_{f}\left(R_{T}\right)}{T}\right)=\operatorname{var} Z
$$

Theorem 1 would be proved if we could change the limit with the variance on the left-hand side. By the dominanting convergence theorem, it is enough to find an integrable majorant for the tails of

$$
X_{T}=\frac{n_{f}\left(R_{T}\right)}{T} \text { and } X_{T}^{2}=\frac{n_{f}\left(R_{T}\right)^{2}}{T^{2}} .
$$

To this end we use the following proposition, which provides uniform exponential bounds on tails of $X_{T}$ :

Proposition 1. Let $f$ be a stationary GAF in some horizontal strip, then using the notation above we have

$$
\exists C, c>0: \sup _{T \geq 1} \mathbb{P}\left(X_{T}>s\right)<C e^{-c s}=h(s) .
$$

Since the proof is quite similar to [7, Prop. 5.1], we defer it to Appendix A. Proposition 1 implies that

$$
\sup _{T \geq 1} \mathbb{P}\left(X_{T}^{2}>s\right)<C e^{-c \sqrt{s}}=h(\sqrt{s}) .
$$


Since both $h(s)$ and $h(\sqrt{s})$ are integrable on $\mathbb{R}$, we have the desired majorants. Exchanging limit and variance then yields:

$$
\lim _{T \rightarrow \infty} \frac{\operatorname{var}\left(n_{f}\left(R_{T}\right)\right)}{T^{2}}=\lim _{T \rightarrow \infty} \operatorname{var}\left(\frac{n_{f}\left(R_{T}\right)}{T}\right)=\operatorname{var}\left(\lim _{T \rightarrow \infty} \frac{n_{f}\left(R_{T}\right)}{T}\right)=\operatorname{var} Z,
$$

and the result is proved.

\section{An Asymptotic Formula for the Variance}

This section is devoted to the derivation of a formula for the variance $V_{f}^{a, b}(T)=\operatorname{var} n_{f}([-T, T] \times$ $[a, b])$ where $T$ is large. We prove the following:

Proposition 2. Let $f$ be a stationary GAF in $D_{\Delta}$ with spectral measure $\rho$. Suppose $\rho$ has no discrete component. Then for any $-\Delta<a<b<\Delta$, and any $T \in \mathbb{R}$, the series

$$
v^{a, b}(T)=\frac{1}{4 \pi^{2}} \sum_{k \geq 1} \frac{1}{k^{2}} \int_{\mathbb{R}} \int_{\mathbb{R}} T \operatorname{sinc}^{2}(2 \pi T(\lambda-\tau)) h_{k}^{a, b}(\lambda+\tau) d \rho^{* k}(\lambda) d \rho^{* k}(\tau)
$$

converges, and

$$
\lim _{T \rightarrow \infty}\left(\frac{V^{a, b}(T)}{2 T}-v^{a, b}(T)\right)=0 .
$$

Here $\rho^{* k}$ is the $k$-fold convolution of $\rho, \operatorname{sinc}(x)=\frac{\sin x}{x}$, and

$$
h_{k}^{a, b}(\lambda)=\left(l_{k}^{a}(\lambda) e^{2 \pi a \lambda}-l_{k}^{b}(\lambda) e^{2 \pi b \lambda}\right)^{2}
$$

where

$$
l_{k}^{y}(\lambda)=\frac{\partial}{\partial y}\left(\frac{1}{r^{k}(2 i y)}\right)+\frac{2 \pi}{r^{k}(2 i y)} \lambda, \text { for } y \in(-\Delta, \Delta), k \in \mathbb{N} .
$$

We begin with some definitions and facts that will be needed along the proof.

\subsection{Preliminaries.}

3.1.1. Tools from Harmonic Analysis. In this section we discuss some operations on measures and their relation to the Fourier transform.

Denote by $\mathcal{M}(\mathbb{R})$ the space of all finite measures on $\mathbb{R}$, similarly $\mathcal{M}^{+}(\mathbb{R})$ denotes all finite non-negative measures on $\mathbb{R}$. For two measure $\mu, \nu \in \mathcal{M}(\mathbb{R})$ the convolution $\mu * \nu \in \mathcal{M}(\mathbb{R})$ is a measure defined by:

$$
\forall \varphi \in C_{0}(\mathbb{R}):(\mu * \nu)(\varphi)=\iint \varphi(\lambda+\tau) d \mu(\lambda) d \nu(\tau)
$$

When both measures have density, this definition agrees with the standard convolution of functions. We write $\mu^{* k}$ for the iterated convolution of $\mu$ with itself $k$ times.

for a measure $\mu \in \mathcal{M}^{+}(\mathbb{R})$ having exponential moments up to $2 \Delta$ (i.e., obeying condition (1)), and a number $y \in(-2 \Delta, 2 \Delta)$, we define the exponentially rescaled measure $\mu_{y} \in \mathcal{M}^{+}(\mathbb{R})$ by

$$
\forall \varphi \in C_{0}(\mathbb{R}): \mu_{y}(\varphi)=\mu\left(e^{2 \pi y \lambda} \varphi(\lambda)\right)=\int_{\mathbb{R}} e^{2 \pi y \lambda} \varphi(\lambda) d \mu(\lambda)
$$


Observation. For any $\mu, \nu \in \mathcal{M}(\mathbb{R})$ and any $|y|<2 \Delta$,

$$
(\mu * \nu)_{y}=\mu_{y} * \nu_{y} .
$$

Proof. for any test function $\varphi \in C_{0}(\mathbb{R})$ we have:

$$
\begin{aligned}
\int \varphi d\left(\mu_{y} * \nu_{y}\right) & =\iint \varphi(\lambda+\tau) d \mu_{y}(\lambda) d \nu_{y}(\tau) \\
& =\iint \varphi(\lambda+\tau) e^{2 \pi y(\lambda+\tau)} d \mu(\lambda) d \nu(\tau)=\int \varphi d(\mu * \nu)_{y}
\end{aligned}
$$

Corollary. If $\rho \in \mathcal{M}^{+}(\mathbb{R})$ is such that (1), then for any $|y|<2 \Delta$ and $k \in \mathbb{N}$ we have $\left(\rho_{y}\right)^{* k}=\left(\rho^{* k}\right)_{y}$, so there will be no ambiguity in the notation $\rho_{y}^{* k}$.

Next, we define for $\mu \in \mathcal{M}(\mathbb{R})$ the flipped measure flip $\{\mu\} \in \mathcal{M}(\mathbb{R})$ by:

$$
\text { flip }\{\mu\}(I)=\mu(-I) \text { for any interval } I \subset \mathbb{R},
$$

and the cross-correlation of measures $\mu, \nu \in \mathcal{M}(\mathbb{R})$ by:

$$
\mu \star \nu:=\mu * \operatorname{flip}\{\nu\} .
$$

An alternative definition, via actions on test-functions, would be:

$$
\forall \varphi \in C_{0}(\mathbb{R}):(\mu \star \nu)(\varphi)=\iint \varphi(\lambda-\tau) d \mu(\lambda) d \nu(\tau)
$$

Notice that the cross-correlation operator is bi-linear, but not commutative. In all following expressions, convolution precedes cross-correlation.

We are now ready to prove a lemma relating these notions to the Fourier tranform.

Lemma 3.1. Suppose $\rho \in \mathcal{M}^{+}(\mathbb{R})$ obeys (1), and $r=\mathcal{F}[\rho]$. Then, for any $|y|<2 \Delta, x \in \mathbb{R}$ and $k \in \mathbb{N}$ :

$$
|r(x+i y)|^{2 k}=\mathcal{F}\left[\rho_{y}^{* k} \star \rho_{y}^{* k}\right](x) .
$$

This measure acts on a test-function $\varphi \in C_{0}(\mathbb{R})$ in the following way:

$$
\left(\rho_{y}^{* k} \star \rho_{y}^{* k}\right)(\varphi)=\iint \varphi(\lambda-\tau) e^{2 \pi y(\lambda+\tau)} d \rho^{* k}(\lambda) d \rho^{* k}(\tau) .
$$

Proof. Fix $k \in \mathbb{N}$. Since $r(z)=\mathcal{F}[\rho](z)$, by a standard property of Fourier transform one has $r^{k}(z)=\mathcal{F}\left[\rho^{* k}\right](z)$. Writing $z=x+i y$, this reads

$$
r^{k}(x+i y)=\int_{\mathbb{R}} e^{-2 \pi i x \lambda} e^{2 \pi y \lambda} d \rho^{* k}(\lambda) .
$$

This implies:

- $r^{k}(x+i y)=\mathcal{F}\left[\rho_{y}^{* k}\right](x)$

- $\overline{r^{k}(x+i y)}=\mathcal{F}\left[\rho_{y}^{* k}\right](-x)=\mathcal{F}\left[\operatorname{flip}\left\{\rho_{y}^{* k}\right\}\right](x)$,

which leads to

$$
|r(x+i y)|^{2 k}=\mathcal{F}\left[\rho_{y}^{* k} * \operatorname{flip}\left\{\rho_{y}^{* k}\right\}\right](x)=\mathcal{F}\left[\rho_{y}^{* k} \star \rho_{y}^{* k}\right](x) .
$$


Also useful to us will be the following special case of Parseval's identity for measures (see Katznelson [12, VI.2.2]):

Lemma 3.2. For any finite measure $\gamma$ on $\mathbb{R}$,

$$
\int_{-2 T}^{2 T}\left(1-\frac{|x|}{2 T}\right) \mathcal{F}[\gamma](x) d x=\int_{\mathbb{R}} 2 T \operatorname{sinc}^{2}(2 \pi T \xi) d \gamma(\xi) .
$$

where $\operatorname{sinc}(\xi)=\frac{\sin \xi}{\xi}$ and $\mathcal{F}[\gamma]$ is the Fourier transform of $\gamma$.

3.1.2. Properties of a "normalized" covariance function. Here we summarize some properties of a normalized version of the covariance function, namely $\frac{|r(x+i a+i b)|^{2}}{r(2 i a) r(2 i b)}$, which will be used later in our proofs. In the following, when we do not specify the variables we mean the statements holds on all the domain of definition. We use the subscript notation for partial derivatives (such as $q_{a}$ for $\frac{\partial}{\partial a} q$ ).

Lemma 3.3. The function

$$
q(x, a, b):=\frac{|r(x+i a+i b)|^{2}}{r(2 i a) r(2 i b)},
$$

is well-defined, infinitely differentiable on $\mathbb{R} \times(-\Delta, \Delta)^{2}$, and satisfies the following properties:

1. $q\left(x, y_{1}, y_{2}\right) \in[0,1]$. $q\left(x, y_{1}, y_{2}\right)=1$ if and only if $\left(x=0\right.$ and $\left.y_{1}=y_{2}\right)$.

2. $\sup _{x \in \mathbb{R}} q\left(x, y_{1}, y_{2}\right)<1$ for any $y_{1} \neq y_{2}$ in $(-\Delta, \Delta)$.

3. For fixed $y_{1}$ and $y_{2}$ let $g_{y_{1}, y_{2}}(x)$ be one of the functions $q, q_{a}, q_{b}, q_{a b}$ evaluated on the line $\left\{\left(x, y_{1}, y_{2}\right): x \in \mathbb{R}\right\}$. Then $g_{y_{1}, y_{2}} \in L^{\infty}(\mathbb{R})$. If condition (2) holds, then for any $y_{1}, y_{2} \in[a, b]$ we have also $g_{y_{1}, y_{2}} \in L^{1}(\mathbb{R}) \cap C_{0}(\mathbb{R})$ (i.e., is integrable and tends to zero as $x \rightarrow \pm \infty)$.

4. $q_{a}(0, t, t)=0$, for any $t \in(-\Delta, \Delta)$.

Proof. Since $r(2 i y)>0$ for all $y \in \mathbb{R}$, the function $q$ is indeed well-defined; differentiability follows from that of $r(z)$.

For item 1, notice that

$$
q(x, a, b)=\frac{\left(\int e^{2 \pi(a+b) \lambda} e^{-2 \pi i x \lambda} d \rho(\lambda)\right)^{2}}{\int e^{2 \pi \cdot 2 a \lambda} d \rho(\lambda) \int e^{2 \pi \cdot 2 a \lambda} d \rho(\lambda)}
$$

and so, by Cauchy-Schwarz, is in $[0,1]$. Equality $q(x, a, b)=1$ holds only if the function $\lambda \mapsto e^{2 \pi \cdot a \lambda} e^{-2 \pi i x \lambda}$ is a constant times the function $\lambda \mapsto e^{2 \pi \cdot b \lambda}, \rho$-a.e., but, if $\rho$ is non-atomic, this is impossible unless $x=0$ and $a=b$.

Further, we notice that

$$
|r(x+i a+i b)|=\left|\int e^{2 \pi(a+b) \lambda} e^{-2 \pi i x \lambda} d \rho(\lambda)\right| \leq \int e^{2 \pi(a+b) \lambda} d \rho(\lambda)=r(i a+i b),
$$

so that $q(x, a, b) \leq q(0, a, b)<1$ (the right-most inequality is by item 1 ). Taking the supremum yields item 2 . 
For item 3, notice any one of the functions $q, q_{a}, q_{b}, q_{a b}$ is the sum of summands of the form

$$
C(a, b) r^{(j)}(x+i a+i b) r^{(m)}(-x+i a+i b),
$$

where $0 \leq j, m \leq 2$ are integers. It is enough therefore to explain why $r^{(j)}(x+i a+i b)$ is bounded and approaches zero as $x \rightarrow \pm \infty$, for any integer $0 \leq j \leq 2$. Recall that

$$
r^{(j)}(x+i y)=c_{j} \mathcal{F}\left[\lambda^{j} e^{2 \pi y \lambda} d \rho(\lambda)\right](x),
$$

where $c_{j}$ is some constant. As a function of $x$, this is a Fourier transform of a non-atomic measure, therefore has the desired properties.

If condition (2) holds, then $d \rho(\lambda)=p(\lambda) d \lambda$, and the function $\lambda \mapsto \lambda^{j} e^{2 \pi\left(y_{1}+y_{2}\right) \lambda} p(\lambda)$ is in $L^{2}(\mathbb{R})$. Then, its Fourier transform $r^{(j)}\left(x+i y_{1}+i y_{2}\right)$ is also in $L^{2}(\mathbb{R})$, and each summand of the form (4) is in $L^{1}(\mathbb{R})$, as anticipated.

For item 4 , notice that for all $x \in \mathbb{R}$ and all $a, b \in(-\Delta, \Delta)$ we have the symmetry $q(x, a, b)=q(x, b, a)$, and therefore for all $t \in \mathbb{R}: q_{a}(x, t, t)=q_{b}(x, t, t)$. On the other hand, for all $t \in(-\Delta, \Delta)$ it holds that $q(0, t, t)=1$, so taking derivative by $t$ we get $q_{a}(0, t, t) \cdot 1+$ $q_{b}(0, t, t) \cdot 1=0$. This proves the result.

We are now ready to begin the proof of Proposition 2,

3.2. Integrals on significant edges. The boundary of the rectangle $R_{T}=[-T, T] \times[a, b]$ is composed of four segments $\partial R_{T}=\bigcup_{1 \leq i \leq 4} I_{j}$ with induced orientation from the counterclockwise orientation of $\partial R_{T}$, where $I_{1}=[-T, T] \times\{a\}$ and $I_{3}=[T,-T] \times\{b\}$. By the argument principle,

$$
n_{f}\left(R_{T}\right)=\sum_{1 \leq i \leq 4} \frac{1}{2 \pi} \triangle_{i}^{T} \arg f
$$

where $\triangle_{i}^{T} \arg f$ is the increment of the argument of $f$ along the segment $I_{i}$ (a.s. $f$ has no zeroes on the boundary of the rectangle $R_{T}$ 1).

Then, by the argument principle,

$$
V_{f}^{a, b}(T)=\operatorname{var}\left[n_{f}\left(R_{T}\right)\right]=\frac{1}{4 \pi^{2}} \sum_{1 \leq i, j \leq 4} \operatorname{cov}\left(\triangle_{i}^{T} \arg f, \triangle_{j}^{T} \arg f\right),
$$

where

$$
\operatorname{cov}(X, Y)=\mathbb{E}[X Y]-\mathbb{E} X \cdot \mathbb{E} Y .
$$

Our first claim is that asymptotically when $T$ is large, the terms involving the (short) vertical segments are negligible in this sum.

\footnotetext{
1 To see this, first notice that the distribution of $n_{f}\left(I_{j}\right)$ for $j=2,4$ (the number of zeroes in a "short" vertical segments) does not depend on $T$. If it were not a.s. zero, then $\mathbb{E} n_{f}\left(I_{2}\right)>0$. Now for any finite set of points $\left\{t_{j}\right\}_{j=1}^{N} \subset[0,1]$, we have $\mathbb{E} n_{f}([0,1] \times[a, b]) \geq \sum_{j=1}^{N} \mathbb{E} n_{f}\left(\left\{t_{j}\right\} \times[a, b]\right)=N \mathbb{E} n_{f}\left(I_{2}\right)$, yielding $\mathbb{E} n_{f}([0,1] \times[a, b])=\infty$ - which is false. For $j=1,3$, recall that since there are no atoms in the spectral measure, $f$ is ergodic with respect to horizontal shifts (this is Fomin-Grenander-Maruyama Theorem, see explanation and references within [7]). This implies that each horizontal line (such as $\left.L_{a}=\mathbb{R} \times\{a\}\right)$ either a.s. contains a zero or a.s. contains no zeroes. If the former holds, then also $\mathbb{E} n_{f}([0,1] \times\{a\})>0$, and the measure $\nu_{f}$ from Theorem $\mathrm{A}$ has an atom at $a$ - contradiction to part (iii) of that Theorem.
} 
Claim 1. As $T \rightarrow \infty$, one has:

$V_{f}^{a, b}(T)=\frac{1}{4 \pi^{2}} \sum_{i, j \in\{1,3\}} \operatorname{cov}\left(\triangle_{i}^{T} \arg f, \triangle_{j}^{T} \arg f\right)+O\left(1+\sqrt{\operatorname{var}\left(\triangle_{1}^{T} \arg f\right)}+\sqrt{\operatorname{var}\left(\triangle_{3}^{T} \arg f\right)}\right)$.

Proof. We demonstrate how to bound one of the terms in (5) involving a "short" vertical segment (corresponding, say, to $i=2$ ). By stationarity, $\operatorname{var}\left(\triangle_{2}^{T} \arg f\right)=\operatorname{var}\left(\triangle_{2}^{0} \arg f\right)=: c^{2}$. Applying the Cauchy-Schwarz inequality, we have:

$$
\begin{aligned}
\operatorname{cov}\left(\triangle_{1}^{T} \arg f, \triangle_{2}^{T} \arg f\right) & \leq \sqrt{\operatorname{var}\left(\triangle_{1}^{T} \arg f\right)} \sqrt{\operatorname{var}\left(\triangle_{2}^{T} \arg f\right)} \\
& =c \cdot \sqrt{\operatorname{var}\left(\triangle_{1}^{T} \arg f\right)} .
\end{aligned}
$$

Let us now give an alternative formulation of Claim 1. Using Cauchy-Riemann equations, we have:

$$
\begin{aligned}
& \triangle_{1}^{T} \arg f=\int_{-T}^{T}\left(\frac{\partial}{\partial x} \arg f(x+i a)\right) d x=-\int_{-T}^{T} \frac{\partial}{\partial a} \log |f(x+i a)| d x=:-X^{a}(T) \\
& \triangle_{3}^{T} \arg f=-\int_{-T}^{T}\left(\frac{\partial}{\partial x} \arg f(x+i b)\right) d x=\int_{-T}^{T} \frac{\partial}{\partial b} \log |f(x+i b)| d x=X^{b}(T)
\end{aligned}
$$

Denoting $C^{a, b}(T)=\operatorname{cov}\left(X^{a}(T), X^{b}(T)\right)$ we may rewrite Claim 1 as

$$
V_{f}^{a, b}(T)=\frac{1}{4 \pi^{2}}\left(C^{a, a}(T)-2 C^{a, b}(T)+C^{b, b}(T)\right)+O\left(1+\sqrt{C^{a, a}(T)}+\sqrt{C^{b, b}(T)}\right),
$$

or alternatively:

Claim 1a. As $T \rightarrow \infty$, we have:

$$
\frac{V^{a, b}(T)}{2 T}=\frac{C^{a, a}(T)-2 C^{a, b}(T)+C^{b, b}(T)}{4 \pi^{2} \cdot 2 T}+O\left(\frac{1+\sqrt{C^{a, a}(T)}+\sqrt{C^{b, b}(T)}}{T}\right) .
$$

where

$$
\begin{aligned}
C^{a, b}(T)=\mathbb{E} & \left\{\int_{-T}^{T} d t \int_{-T}^{T} d s\left(\frac{\partial}{\partial a} \log |f(t+i a)| \frac{\partial}{\partial b} \log |f(s+i b)|\right)\right\} \\
& -\mathbb{E}\left\{\int_{-T}^{T} \frac{\partial}{\partial a} \log |f(t+i a)| d t\right\} \mathbb{E}\left\{\int_{-T}^{T} \frac{\partial}{\partial b} \log |f(s+i b)| d s\right\}
\end{aligned}
$$

3.3. Changing order of operations. Our goal now is to prove the following:

\section{Claim 2.}

$$
C^{a, b}(T)=\int_{-T}^{T} \int_{-T}^{T} \frac{\partial^{2}}{\partial a \partial b} \operatorname{cov}(\log |f(t+i a)|, \log |f(s+i b)|) d t d s .
$$

The meaning of this formula for $C^{a, a}(T)$ is as follows: on the RHS, first take the mixed partial derivative (as if $a \neq b$ ), then substitute $b=a$ and integrate by $t$ and $s$.

The proof is an application of the following two lemmas. In both, we assume $f$ is a stationary GAF in $D_{\Delta}$, and $a, b \in(-\Delta, \Delta)$. 
Lemma 3.4. For any $T>0$ the following integrals are finite:

$$
\begin{aligned}
& \int_{-T}^{T} \mathbb{E}\left|\frac{f^{\prime}(t+i a)}{f(t+i a)}\right| d t<\infty . \\
& \int_{-T}^{T} \int_{-T}^{T} \mathbb{E}\left|\frac{f^{\prime}(t+i a)}{f(t+i a)} \frac{f^{\prime}(s+i b)}{f(s+i b)}\right| d t d s<\infty .
\end{aligned}
$$

Lemma 3.5. For almost all $t, s \in[-T, T]^{2}$,

$$
\begin{aligned}
& \mathbb{E}\left[\frac{\partial}{\partial a} \log |f(t+i a)|\right]=\frac{\partial}{\partial a} \mathbb{E}[\log |f(t+i a)|] \\
& \mathbb{E}\left[\frac{\partial^{2}}{\partial a \partial b} \log |f(t+i a)| \log |f(s+i b)|\right]=\frac{\partial^{2}}{\partial a \partial b} \mathbb{E}[\log |f(t+i a)| \log |f(s+i b)|] .
\end{aligned}
$$

Though simple to state, Lemmas 3.4 and 3.5 both require long and non-trivial proofs, which do not follow any standard method we are aware of. We therefore defer their proofs to Appendix B.

Proof of Claim [. Recall the definition of $C^{a, b}(T)$ in ([6), and notice that

$$
\left|\frac{\partial}{\partial a} \log \right| f(x+i a)|| \leq\left|\frac{f^{\prime}(x+i a)}{f(x+i a)}\right| .
$$

Step 1: Exchange integrals and expcectation: By (8) and Lemma 3.4, we may apply Fubini's theorem to get:

$$
\begin{aligned}
C^{a, b}(T)=\int_{-T}^{T} \int_{-T}^{T}\left[\mathbb{E} \frac{\partial^{2}}{\partial a \partial b}\{\log |f(t+i a)| \log |f(s+i b)|\}\right. & \\
- & \left.\mathbb{E} \frac{\partial}{\partial a} \log |f(t+i a)| \mathbb{E} \frac{\partial}{\partial b} \log |f(s+i b)|\right] d t d s .
\end{aligned}
$$

Step 2: Exchage the order of expectation and derivative inside the integral by $t$ and $s$. This is justified directly by Lemma 3.5. We arrive at the desired form.

3.4. The error term. Next, we show that the error term in Claim 1a approaches zero as $T$ tends to infinity.

Claim 3. If $\rho$ contains no atoms, then for any $a \in(-\Delta, \Delta)$ :

$$
\lim _{T \rightarrow \infty} \frac{C^{a, a}(T)}{T^{2}}=0
$$

Proof. Since $\rho$ has no atoms, $f$ is an ergodic process (this is the classical Fomin-GrenanderMaruyama theorem, see [7, Theorem 4] and references therein). Thus, by the ergodic theorem,

$$
\lim _{T \rightarrow \infty} \frac{1}{T} X^{a}(T)=\mathbb{E} X^{a}(1)
$$

converges almost surely and in $L^{1}$ to a constant. Recall $X^{a}(T)$ has finite second moment (this is precisely relation (A-II) $)$. Therefore, the convergence in (9) is also in the $L^{2}$ sense (this 
is an easy adaptation of the proof for $L^{1}$ convergence, see [6, Exercise 7.2.1]). We conclude that:

$$
\lim _{T \rightarrow \infty} \frac{1}{T^{2}} \operatorname{var}\left(X^{a}(T)\right)=\lim _{T \rightarrow \infty} \frac{1}{T^{2}} C^{a, a}(T)=0 .
$$

Claims 1a, 2 and 3 give together:

\section{Corollary.}

$$
\frac{V^{a, b}(T)}{2 T}=\frac{C^{a, a}(T)-2 C^{a, b}(T)+C^{b, b}(T)}{4 \pi^{2} \cdot 2 T}+o(1), \quad T \rightarrow \infty,
$$

where $C^{a, b}(T)$ is given by (7).

3.5. A formula in terms of the covariance function. Our goal now is to replace (7) by a simpler formula, using the covariance function. This is done in the next claim.

\section{Claim 4.}

$$
\frac{C^{a, b}(T)}{2 T}=\frac{1}{2} \int_{-2 T}^{2 T}\left(1-\frac{|x|}{2 T}\right) \sum_{k \geq 1} \frac{\partial^{2}}{\partial a \partial b} \frac{q(x, a, b)^{k}}{k^{2}} d x
$$

where

$$
q(x, a, b):=\frac{|r(x+i a+i b)|^{2}}{r(2 i a) r(2 i b)} .
$$

For the proof, we will need the following Lemma, which is a direct consequence of a lemma by Nazarov and Sodin [14, Lemma 2.2] (see also [10, Lemma 3.5.2]).

Lemma 3.6. If $\xi$ and $\eta$ are centered complex Gaussian random variables, then:

$$
\operatorname{cov}(\log |\xi|, \log |\eta|)=\frac{1}{4} \sum_{k=1}^{\infty} \frac{1}{k^{2}}\left(\frac{|\mathbb{E}(\xi \bar{\eta})|^{2}}{\mathbb{E}|\xi|^{2} \mathbb{E}|\eta|^{2}}\right)^{k} .
$$

Proof of Claim 4. Taking $\xi=f(t+i a)$ and $\eta=f(s+i b)$, we have due to stationarity:

$$
\frac{|\mathbb{E}(\xi \bar{\eta})|^{2}}{\mathbb{E}|\xi|^{2} \mathbb{E}|\eta|^{2}}=\frac{|\mathbb{E}(f(t+i a) \overline{f(s+i b)})|^{2}}{\mathbb{E}|f(t+i a)|^{2} \mathbb{E}|f(s+i b)|^{2}}=\frac{|r(t-s+i a+i b)|^{2}}{r(2 i a) r(2 i b)}=q(t-s, a, b) .
$$

Therefore, by Lemma 3.6 equation (77) becomes:

$$
\begin{aligned}
C^{a, b}(T) & =\frac{1}{4} \int_{-T}^{T} \int_{-T}^{T}\left\{\frac{\partial^{2}}{\partial a \partial b} \sum_{k=1}^{\infty} \frac{1}{k^{2}} q(t-s, a, b)^{k}\right\} d t d s \\
& =\frac{1}{2} \int_{-2 T}^{2 T}\left(1-\frac{|x|}{2 T}\right)\left\{\frac{\partial^{2}}{\partial a \partial b} \sum_{k \geq 1} \frac{q(x, a, b)^{k}}{k^{2}}\right\} d x .
\end{aligned}
$$

In the last equality we used that $\int_{-T}^{T} \int_{-T}^{T} Q(t-s) d t d s=2 \int_{-2 T}^{2 T}(2 T-|x|) Q(x) d x$ for any $\left.Q \in L^{1}([-2 T, 2 T])\right)$, which can be proved by a simple change of variables. 
All that remains in order to get (11), is to prove that

$$
\forall x \neq 0, \quad \frac{\partial^{2}}{\partial a \partial b} \sum_{k \geq 1} \frac{q(x, a, b)^{k}}{k^{2}}=\sum_{k \geq 1} \frac{\partial^{2}}{\partial a \partial b} \frac{q(x, a, b)^{k}}{k^{2}}
$$

Fix $x \neq 0$. For shortness, we do not write the variables $(x, a, b)$, and use again the subscript notation for partial derivatives. We compute:

$$
S_{k}^{a, b}(x):=\frac{\partial^{2}}{\partial a \partial b}\left\{q^{k}\right\}= \begin{cases}q_{a b}, & k=1 \\ k(k-1) q^{k-2} q_{a} q_{b},+k q^{k-1} q_{a b} & k>1\end{cases}
$$

Therefore,

$$
\left|\frac{S_{k}^{a, b}(x)}{k^{2}}\right| \leq q^{k-2}\left|q_{a} q_{b}\right|+\frac{1}{k} q^{k-1}\left|q_{a b}\right| .
$$

By part 1 of Lemma 3.3, $q(x, a, b)<1$ (notice this holds also if $a=b$ ). We deduce that $\sum\left|\frac{S_{k}^{a, b}}{k^{2}}\right|<\infty$. i.e., the RHS of (13) converges in absolute value. By standard arguments, this is enough to prove equality (13). Claim 4 follows.

3.6. A formula in terms of the spectral measure. In this section, we finally prove Proposition 2, by carefully moving to the spectral representation of the formula we had at hand.

Proof of Proposition 2. Using Lemma 3.1 and the definition of $q$ in (12), we get

$$
q(x, a, b)^{k}=\frac{\mathcal{F}\left[\rho_{a+b}^{* k} \star \rho_{a+b}^{* k}\right](x)}{r^{k}(2 i a) r^{k}(2 i b)}
$$

Define

$$
S_{k}^{a, b}(x):=\frac{\partial^{2}}{\partial a \partial b}\left\{q(x, a, b)^{k}\right\}=\frac{\partial^{2}}{\partial a \partial b}\left\{\frac{\mathcal{F}\left[\rho_{a+b}^{* k} \star \rho_{a+b}^{* k}\right](x)}{r^{k}(2 i a) r^{k}(2 i b)}\right\} .
$$

\section{Observation.}

$$
S_{k}^{a, b}(x)=\mathcal{F}\left[l_{k}^{a}(\lambda) \rho_{a+b}^{* k} \star l_{k}^{b}(\lambda) \rho_{a+b}^{* k}\right](x) .
$$

where $l_{k}^{a}(\lambda), l_{k}^{b}(\lambda)$ are linear functions in $\lambda$, given by

$$
l_{k}^{a}(\lambda)=\frac{\partial}{\partial a}\left(\frac{1}{r^{k}(2 i a)}\right)+\frac{2 \pi}{r^{k}(2 i a)} \lambda=\frac{2}{r^{k}(2 i a)}\left(-i k \frac{r^{\prime}(2 i a)}{r(2 i a)}+\pi \lambda\right) .
$$

Proof. Recall that

$$
\mathcal{F}\left[\rho_{a+b}^{* k} \star \rho_{a+b}^{* k}\right](x)=\iint e^{-i x(\lambda-\tau)} e^{2 \pi i(a+b)(\lambda+\tau)} d \rho^{* k}(\lambda) d \rho^{* k}(\tau),
$$

and notice we may differentiate by $a$ and $b$ under the integral, as the result would be continuous and integrable w.r.t. $\rho^{* k}$. From here, the proof is a straightforward computation. 
Using this observation, we rewrite equation (11) as follows:

$$
\frac{C^{a, b}(T)}{2 T}=\int_{-2 T}^{2 T}\left(1-\frac{|x|}{2 T}\right) \sum_{k \geq 1} \frac{1}{2 k^{2}} \frac{\partial^{2}}{\partial a \partial b}\left\{\frac{\mathcal{F}\left[\rho_{a+b}^{* k} \star \rho_{a+b}^{* k}\right](x)}{r^{k}(2 i a) r^{k}(2 i b)}\right\} d x .
$$

Futher, using Lemma 3.2 (Parseval's identity), we have for fixed $k$,

$$
\begin{gathered}
\int_{-2 T}^{2 T}\left(1-\frac{|x|}{2 T}\right) S_{k}^{a, b}(x) d x=\int_{-2 T}^{2 T}\left(1-\frac{|x|}{2 T}\right) \mathcal{F}\left[l_{k}^{a}(\lambda) \rho_{a+b}^{* k} \star l_{k}^{b}(\lambda) \rho_{a+b}^{* k}\right](x) \\
=\int_{\mathbb{R}} \int_{\mathbb{R}} 2 T \operatorname{sinc}^{2}(2 \pi T(\lambda-\tau)) l_{k}^{a}(\lambda) l_{k}^{b}(\tau) e^{2 \pi(a+b)(\lambda+\tau)} d \rho^{* k}(\lambda) d \rho^{* k}(\tau) .
\end{gathered}
$$

Plugging (15) into (10), we get:

$$
\begin{aligned}
\frac{V_{f}^{a, b}(T)}{2 T}= & \frac{1}{4 \pi^{2}} \int_{-2 T}^{2 T}\left(1-\frac{|x|}{2 T}\right) \sum_{k \geq 1} \frac{1}{2 k^{2}}\left(S_{k}^{a, a}(x)-2 S_{k}^{a, b}(x)+S_{k}^{b, b}(x)\right) d x+o(1) \\
& =\frac{1}{4 \pi^{2}} \sum_{k \geq 1} \frac{1}{2 k^{2}} \int_{-2 T}^{2 T}\left(1-\frac{|x|}{2 T}\right)\left(S_{k}^{a, a}(x)-2 S_{k}^{a, b}(x)+S_{k}^{b, b}(x)\right) d x+o(1) \\
& =\frac{1}{8 \pi^{2}} \sum_{k \geq 1} \frac{1}{k^{2}} \int_{\mathbb{R}} \int_{\mathbb{R}} T \operatorname{sinc}^{2}(2 \pi T(\lambda-\tau)) h_{k}^{a, b}(\lambda+\tau) d \rho^{* k}(\lambda) d \rho^{* k}(\tau)+o(1),
\end{aligned}
$$

where

$$
h_{k}^{a, b}(\lambda)=\left(l_{k}^{a}(\lambda) e^{2 \pi a \lambda}-l_{k}^{b}(\lambda) e^{2 \pi b \lambda}\right)^{2} .
$$

The exchange of sum and integral in the second equality of (17) is justified by the monotone convergence theorem, as each term in the series is non-negative. The last equality follows from (16). Equation 17 establishes Proposition 2 .

\section{Theorem 2; Linear and Intermediate Variance}

We dedicate Section 4.1 to prove some facts which will be needed along the proof. Afterwards, we prove the existence of the limit $L_{1}$ and its positivity in Section 4.2 . In Section 4.3 we prove $L_{1}$ is finite under condition (2).

\subsection{Preparation.}

4.1.1. Tools from Analysis. First we present two observations about convolutions and integration.

Observation 4.1. If $Q: \mathbb{R} \rightarrow[0, \infty)$ is integrable on $\mathbb{R}$, then

$$
\lim _{T \rightarrow \infty} \int_{-T}^{T}\left(1-\frac{|x|}{T}\right) Q(x) d x=\int_{\mathbb{R}} Q .
$$

Proof. Notice that:

$$
\int_{-\sqrt{T}}^{\sqrt{T}}\left(1-\frac{1}{\sqrt{T}}\right) Q(x) d x \leq \int_{-T}^{T}\left(1-\frac{|x|}{T}\right) Q(x) d x \leq \int_{-T}^{T} Q(x) d x,
$$

and both ends of the inequality approach the limit $\int_{\mathbb{R}} Q$. 
Observation 4.2. For any $\psi_{1}, \psi_{2} \in C_{0}(\mathbb{R})$ and $\mu \in \mathcal{M}^{+}(\mathbb{R})$,

$$
\int \psi_{1} d\left(\mu * \psi_{2}\right)=\int\left(\psi_{1} * \operatorname{fip}\left\{\psi_{2}\right\}\right) d \mu
$$

Proof.

$$
\begin{aligned}
& \int \psi_{1} d\left(\mu * \psi_{2}\right)=\int \psi_{1}(x+y) d \mu(x) \psi_{2}(y) d y \\
& \quad=\int\left(\int \psi_{1}(x+y) \operatorname{fiip}\left\{\psi_{2}\right\}(-y) d y\right) d \mu(x)=\int\left(\psi_{1} * \operatorname{flip}\left\{\psi_{2}\right\}\right)(x) d \mu(x) .
\end{aligned}
$$

The following lemma shall play a key-role later on in our proof.

Lemma 4.1. Let $\mu \in \mathcal{M}^{+}(\mathbb{R})(\mu \neq \equiv 0)$. Then the following limit exists (finite or infinite):

$$
\lim _{\varepsilon \rightarrow 0+} \int_{\mathbb{R}} \frac{1}{2 \varepsilon} \mu(\tau-\varepsilon, \tau+\varepsilon) d \mu(\tau)=\int_{\mathbb{R}}|\mathcal{F}[\mu]|^{2}(x) d x .
$$

Proof. Denote $\varphi_{\varepsilon}=\frac{1}{2 \varepsilon} \mathbb{I}_{(-\varepsilon, \varepsilon)}$ for $\varepsilon>0$. Rewriting the integral and using Parseval's identity, we get:

$$
\begin{aligned}
I_{\mu}(\varepsilon) & :=\frac{1}{2 \varepsilon} \int_{\mathbb{R}} \mu(\tau-\varepsilon, \tau+\varepsilon) d \mu(\tau) \\
& =\int_{\mathbb{R}}\left(\mu * \varphi_{\varepsilon}\right)(\tau) d \mu(\tau) \\
& =\int_{\mathbb{R}}\left(\mathcal{F}[\mu] \cdot \mathcal{F}\left[\varphi_{\varepsilon}\right]\right)(x) \mathcal{F}[\mu](-x) d x \\
& =\int_{\mathbb{R}} \operatorname{sinc}(2 \pi \varepsilon x)|\mathcal{F}[\mu]|^{2}(x) d x
\end{aligned}
$$

Since $|\operatorname{sinc}(2 \pi \varepsilon x)| \leq 1$, we have the upper bound $I_{\mu}(\varepsilon) \leq \int_{\mathbb{R}}|\mathcal{F}[\mu]|^{2}(x) d x$.

Using the Observation 4.2 and the fact that $\varphi_{\varepsilon} * \varphi_{\varepsilon} \leq 2 \varphi_{2 \varepsilon}$ we get:

$$
\int_{\mathbb{R}}\left(\mu * \varphi_{\varepsilon}\right) d\left(\mu * \varphi_{\varepsilon}\right)=\int_{\mathbb{R}} \mu *\left(\varphi_{\varepsilon} * \varphi_{\varepsilon}\right) d \mu \leq 2 \int_{\mathbb{R}} \mu * \varphi_{2 \varepsilon} d \mu=2 I_{\mu}(2 \varepsilon)
$$

On the other hand,

$$
\begin{aligned}
\int_{\mathbb{R}}\left(\mu * \varphi_{\varepsilon}\right) d\left(\mu * \varphi_{\varepsilon}\right) & =\int\left|\mathcal{F}\left[\mu * \varphi_{\varepsilon}\right]\right|^{2}=\int|\mathcal{F}[\mu]|^{2} \cdot \operatorname{sinc}^{2}(2 \pi \varepsilon x) d x \\
& \geq \int_{K}|\mathcal{F}[\mu]|^{2} \cdot \operatorname{sinc}^{2}(2 \pi \varepsilon x) d x
\end{aligned}
$$

for any compact set $K \subset \mathbb{R}$. Since the limit $\lim _{\varepsilon \rightarrow 0+} \operatorname{sinc}(2 \pi \varepsilon x)=1$ is uniform in $x \in K$, the last expression approaches $\int_{K}|\mathcal{F}[\mu]|^{2}$ as $\varepsilon \rightarrow 0+$. Thus, by choosing $K$ and then $\varepsilon>0$ properly, the lower bound may be made arbitrarily close to $\int_{\mathbb{R}}|\mathcal{F}[\mu]|^{2}$. This concludes the proof. 
4.1.2. Lower bounds on $h_{1}^{a, b}$. The main goal of this subsection is to give lower bounds on $h_{1}^{a, b}$ (defined in (18) ). We begin with a simple claim.

Claim 5. The function $h_{1}^{a, b}$ has exactly two real zeroes.

Proof. By the form of $h_{1}^{a, b}, h_{1}^{a, b}(\lambda)=0$ if and only if

$$
e^{2 \pi(b-a) \lambda}=\frac{l_{1}^{a}(\lambda)}{l_{1}^{b}(\lambda)}=\frac{\frac{1}{r(2 i a)}\left(\pi \lambda-i \frac{r^{\prime}}{r}(2 i a)\right)}{\frac{1}{r(2 i b)}\left(\pi \lambda-i \frac{r^{\prime}}{r}(2 i b)\right)}=C \cdot \frac{\lambda-\psi(a)}{\lambda-\psi(b)},
$$

where $C>0$ is a positive constant and $\psi(y)=\frac{1}{2 \pi} \frac{d}{d y}[\log r(2 i y)]$. Since $y \mapsto \log r(2 i y)$ is a convex function, for $a<b$ we have $\psi(a)<\psi(b)$. Therefore, $\lambda \mapsto C \frac{\lambda-\psi(a)}{\lambda-\psi(b)}$ is a strictly decreasing function, with a pole at $\psi(b)$ and with the same positive limit at $\pm \infty$. Thus, it crosses exactly twice the increasing exponential function $e^{2 \pi(b-a) \lambda}$.

The next claim will enable us to bound $h_{1}^{a, b}$ from below, on most of the real line. Denote by $z_{1}, z_{2} \in \mathbb{R}\left(z_{1}<z_{2}\right)$ the two real zeroes of $h_{1}^{a, b}$ whose existence is guaranteed by Claim 5 , We also use the notation $B(x, \delta)$ for the interval of radius $\delta>0$ around $x \in \mathbb{R}$.

Claim 6. For all $\delta>0$, there exists $c_{\delta}>0$ such that for all $\lambda \in \mathbb{R} \backslash\left(B\left(z_{1}, \delta\right) \cup B\left(z_{2}, \delta\right)\right)$ :

$$
h_{1}^{a, b}(\lambda)>c_{\delta}\left(1+\lambda^{2}\right) \max \left(e^{2 a \cdot 2 \pi \lambda}, e^{2 b \cdot 2 \pi \lambda}\right) .
$$

Proof. Since the function $\frac{h_{1}^{a, b}(\lambda)}{\left(1+\lambda^{2}\right) e^{2 a \cdot 2 \pi \lambda}}=\left(\frac{l_{1}^{a}(\lambda)-l_{1}^{b}(\lambda) e^{2 \pi(b-a) \lambda}}{\sqrt{1+\lambda^{2}}}\right)^{2}$ approaches strictly positive limits as $|\lambda| \rightarrow \infty$, there exist $M_{a}, c_{a}>0$ such that

$$
\forall|\lambda| \geq M_{a}: h_{1}^{a, b}(\lambda) \geq c_{a}\left(1+\lambda^{2}\right) e^{2 a \cdot 2 \pi \lambda} .
$$

Similarly, there exist some $M_{b}, c_{b}>0$ such that $\forall|\lambda| \geq M_{b}: h_{1}^{a, b}(\lambda) \geq c_{b}\left(1+\lambda^{2}\right) e^{2 b \cdot 2 \pi \lambda}$. Take $M=\max \left(M_{a}, M_{b}\right)$. Since $h(\lambda)$ attains a positive minimum on $[-M, M] \backslash\left(B\left(z_{1}, \delta\right) \cup B\left(z_{2}, \delta\right)\right)$, there exists some $c>0$ such that for all $\lambda$ in this set, $h(\lambda) \geq c\left(1+\lambda^{2}\right) \max \left(e^{2 a \cdot 2 \pi \lambda}, e^{2 b \cdot 2 \pi \lambda}\right)$. Choosing $c_{\delta}=\min \left(c, c_{a}, c_{b}\right)$ will yield the result.

The next claim is a slight modification of the previous one, in order to fit our specific need. Denote $\operatorname{Diag}_{\varepsilon}=\mathbb{I}\{(\lambda, \tau):|\lambda-\tau|<\varepsilon\}$.

Claim 7. For every $\delta>0$ there exist a set $F=F_{\delta}=\mathbb{R} \backslash\left(I_{1} \cup I_{2}\right)$ such that $I_{j}$ is an interval containing $z_{j}$ and of length at most $\delta(j=1,2), \rho(F)>0$, and there exists $c_{\delta}>0$ such that for all small enough $\varepsilon$,

$$
h(\lambda+\tau) \geq c_{\delta}\left(1+(\lambda+\tau)^{2}\right) \max \left(e^{2 a \cdot 2 \pi(\lambda+\tau)}, e^{2 b \cdot 2 \pi(\lambda+\tau)}\right),
$$

for all $\lambda, \tau \in(F \times F) \cap \operatorname{Diag}_{\varepsilon}$.

Proof. Choose $F=\mathbb{R} \backslash\left(B\left(\frac{z_{1}}{2}, \delta_{0}\right) \cup B\left(\frac{z_{2}}{2}, \delta_{0}\right)\right)$, where $\delta_{0} \leq \delta$ is small enough so that $\rho(F)>$ 0 . Then, for $\varepsilon \leq \delta_{0}$ and $(\lambda, \tau) \in(F \times F) \cap \operatorname{Diag}_{\varepsilon}$, we have

$$
\left|\lambda+\tau-z_{j}\right| \geq\left|2 \tau-z_{j}\right|-|\lambda-\tau| \geq 2 \delta_{0}-\varepsilon \geq \delta_{0} .
$$


Choosing the constant $c_{\delta}>0$ which is the consequence of applying Claim [6 will end our proof.

4.1.3. Convergence properties of the functions $S_{k}$. Recall the definition of $S_{k}^{a, b}$ in (14). We stress, once again, that $S_{k}^{a, a}(x)$ denotes the evaluation of the same mixed partial derivative at the point $(x, a, a)$. Our goal in this subsection is to prove the following Lemma.

Lemma 4.2. If condition (2) is satisfied, then for every $k \in \mathbb{N}$ the functions $S_{k}^{a, a}(x), S_{k}^{a, b}(x)$ and $S_{k}^{b, b}(x)$ are in $L^{1}(\mathbb{R})$ with respect to the variable $x$. Moreover,

$$
\sum_{k \geq 1} \frac{1}{k^{2}} \int_{\mathbb{R}} S_{k}(x) d x \text { converges, }
$$

with any of the three possible superscripts on the letter $S$.

We will need some convergence properties of the function $q$ and its partial derivatives. The first is simple.

Observation 4.3. Let $g$ be one of the functions $q, q_{a}, q_{b}$ or $q_{a b}$. Then $g(x, a, a), g(x, a, b)$ and $g(x, b, b)$ are all in $\left(L^{1} \cap L^{\infty}\right)(\mathbb{R})$ with respect to the variable $x$.

Proof. Follows from part 3 of Lemma 3.3.

The next two claims require some more effort.

Claim 8. The sum $\sum_{m \geq 1} \int_{\mathbb{R}} q^{m} q_{a} q_{b} d x$ converges.

Proof. We will show, in fact, that the positive series $\sum_{m \geq 1} \int_{\mathbb{R}} q^{m}\left|q_{a} q_{b}\right| d x$ converges.

First, in case we are evaluating at $(x, a, b)(a<b)$, our series converges due to (21) and the bound in part 2 of Lemma 3.3. Now assume we are evaluating at $(x, t, t)$ (where $t \in\{a, b\}$ ). As we deal with a positive series, it is enough to show that both

(I) $\quad \sum_{m \geq 1} \int_{-1}^{1} q^{m}\left|q_{a} q_{b}\right| d x<\infty$, and

(II) $\quad \sum_{m \geq 1} \int_{|x| \geq 1} q^{m}\left|q_{a} q_{b}\right| d x<\infty$.

Denote by $C=\sup _{x \in \mathbb{R}}\left|q_{a} q_{b}(x, t, t)\right| \in(0, \infty)$. The sum in (II) is bounded by

$$
C \sum_{m \geq 1} \int_{|x| \geq 1} q^{m}(x, t, t) d x=C \int_{|x| \geq 1} \frac{q}{1-q}(x, t, t) d x \leq C^{\prime} \int_{\mathbb{R}} q(x, t, t) d x,
$$

where $C^{\prime} \in(0, \infty)$ is another constant. $C, C^{\prime}$ and $\int_{\mathbb{R}} q(x, t, t) d x$ are all finite by part 3 of Lemma 3.3 .

We turn to show $(\mathrm{I})$, By parts 1 and 4 of Lemma 3.3, the sum

$$
\sum_{m \geq 1} q^{m}\left|q_{a} q_{b}\right| d x=\frac{\left|q_{a} q_{b}\right|}{1-q}
$$

is well-defined for all $x$ (including $x=0$ ). By the monotone convergence theorem, item $(\mathrm{I})$ is then equivalent to

$$
\int_{-1}^{1} \frac{\left|q_{a} q_{b}\right|}{1-q}(x, t, t) d x<\infty
$$


which is indeed finite as an integral of a continuous function on $[-1,1]$.

Claim 9. The sum $\sum_{m \geq 1} \frac{1}{m+2} \int_{\mathbb{R}} q^{m} d x$ converges.

Proof. We use a fact which appears in standard proofs of the Central Limit Theorem (CLT). For completeness, we include a proof in appendix C.

Lemma 4.3. Let $g \in L^{1}(\mathbb{R})$ be a probability density, i.e., $g \geq 0$ and $\int_{\mathbb{R}} g=1$. Suppose further that

(a) $\quad \int_{\mathbb{R}}|\lambda|^{k} g(\lambda) d \lambda<\infty$ for $k=1,2,3$ and

(b) $\quad \int_{\mathbb{R}}|\mathcal{F}[g](x)|^{\nu} d x<\infty$ for some $\nu \geq 1$.

Then there exists $C>0$ such that for all $m \geq \nu$,

$$
\int_{\mathbb{R}}|\mathcal{F}[g](x)|^{m} d x<\frac{C}{\sqrt{m}} .
$$

We would like to apply Lemma 4.3 to

$$
g^{a, b}(\lambda)=\frac{e^{2 \pi(a+b) \lambda} p(\lambda)}{r(i a+i b)} .
$$

Notice that this is the density of a probability measure. This choice also obeys the extra integrability conditions in the lemma (Condition (a) follows from the exponential moment assumption (11), and condition (b) with $\nu=2$ follows from the $L^{2}$ assumption (2)). We see now that

$$
q(x, a, b)=\frac{r(i a+i b)^{2}}{r(2 i a) r(2 i b)} \cdot\left|\mathcal{F}\left[g^{a, b}\right](x)\right|^{2} \leq\left|\mathcal{F}\left[g^{a, b}\right](x)\right|^{2},
$$

the last inequality following from the log-convexity of $y \mapsto r(i y)$. Similarly we define $g^{a, a}$ and have $q(x, a, a)=\left|\mathcal{F}\left[g^{a, a}\right](x)\right|^{2}$. Thus in all three cases of evaluation, using the lemma with the appropriate function $g$ yields:

$$
\begin{aligned}
\sum_{m \geq 1} \frac{1}{m+2} \int_{\mathbb{R}} q^{m} d x & \leq \sum_{m \geq 1} \frac{1}{m+2} \int_{\mathbb{R}}|\mathcal{F}[g](x)|^{2 m} d x \\
& <C \sum_{m \geq 1} \frac{1}{(m+2) \sqrt{2 m}}<\infty
\end{aligned}
$$

as required.

Proof of Lemma 4.2. Taking derivative by the chain rule, we see that:

$$
S_{k}=\frac{\partial^{2}}{\partial a \partial b}\left\{q^{k}\right\}= \begin{cases}q_{a b}, & k=1 \\ k(k-1) q^{k-2} q_{a} q_{b},+k q^{k-1} q_{a b} & k>1 .\end{cases}
$$

Now, the fact that $S_{k}^{a, a}, S_{k}^{a, b}$ and $S_{k}^{b, b}$ are in $L^{1}(\mathbb{R})$ with respect to $x$ follows from Observation 4.3 . 
We turn now to prove the "moreover" part of the claim. We use (19) in order to rewrite the desired series:

$$
\begin{aligned}
& \sum_{k \geq 1} \frac{1}{k^{2}} \int_{\mathbb{R}} S_{k}(x) d x \\
& \quad=\int_{\mathbb{R}} q_{a b} d x+\sum_{k \geq 2} \int_{\mathbb{R}} q^{k-2} q_{a} q_{b} d x+\sum_{k \geq 2} \frac{1}{k} \int_{\mathbb{R}} q^{k-2}\left(q q_{a b}-q_{a} q_{b}\right) d x .
\end{aligned}
$$

Once again, all functions are evaluated at $(x, a, a),(x, a, b)$ or $(x, b, b)$ and what follows holds for each of the three options. By Observation 4.3 .

$$
\int_{\mathbb{R}}|q| d x<\infty, \quad \int_{\mathbb{R}}\left|q_{a b}\right| d x<\infty, \quad \int_{\mathbb{R}}\left|q_{a} q_{b}\right| d x<\infty
$$

and in particular the left-most term in (20) is finite. For the middle sum in (20), convergence follows from Claim 8 and (21). Convergence of the right-most sum in (20) follows from Claim 9 and (21). This ends the proof of Lemma 4.2 .

4.2. Existence and Positivity. In this section we prove that $L_{1}$ exists and belongs to $(0, \infty]$. If $\rho$ has at least one atom, Theorem 1 implies that $\lim _{T \rightarrow \infty} \frac{V_{f}^{a, b}(T)}{T^{2}}>0$, and therefore $L_{1}=\lim _{T \rightarrow \infty} \frac{V_{f}^{a, b}(T)}{T}=\infty$. We thus assume that $\rho$ has no atoms.

Using the formula for the variance obtained in Proposition 2, and recalling the functions $h_{k}^{a, b}$ are non-negative, we see that the limit $L_{1}$ exists and is in $[0, \infty]$. More effort is needed in order to establish that $L_{1}>0$. We begin with a simple bound arising from Proposition 2 ;

$$
\begin{aligned}
\liminf _{T \rightarrow \infty} \frac{V_{f}^{a, b}(T)}{2 T} & =\frac{1}{4 \pi^{2}} \liminf _{T \rightarrow \infty} \sum_{k \geq 1} \frac{1}{k^{2}} \int_{\mathbb{R}} \int_{\mathbb{R}} T \operatorname{sinc}^{2}(2 \pi T(\lambda-\tau)) h_{k}^{a, b}(\lambda+\tau) d \rho^{* k}(\lambda) d \rho^{* k}(\tau) \\
& \geq \frac{1}{4 \pi^{2}} \liminf _{T \rightarrow \infty} \int_{\mathbb{R}} \int_{\mathbb{R}} T \operatorname{sinc}^{2}(2 \pi T(\lambda-\tau)) h_{1}^{a, b}(\lambda+\tau) d \rho(\lambda) d \rho(\tau) \\
& \geq C_{0} \liminf _{\varepsilon \rightarrow 0+} \int_{\mathbb{R}} \int_{\mathbb{R}} \frac{1}{2 \varepsilon} \mathbb{I}\{(\lambda, \tau):|\lambda-\tau|<\varepsilon\} h_{1}^{a, b}(\lambda+\tau) d \rho(\lambda) d \rho(\tau),
\end{aligned}
$$

where $C_{0}>0$ is an absolute constant. the first inequality follows from considering only the first term in the original non-negative series. The second inequality follows by noticing that $T \operatorname{sinc}^{2}(2 \pi T \lambda) \geq \frac{4 T}{\pi^{2}} \mathbb{I}\left\{\lambda:|\lambda|<\frac{1}{4 T}\right\}$.

Fix a parameter $\delta>0$, and fix $F=F_{\delta}$ to be the set provided by Claim 7 . Continuing (22), we have

$$
\begin{aligned}
\liminf _{T \rightarrow \infty} \frac{V_{f}^{a, b}(T)}{2 T} & \geq c_{\delta} \liminf _{\varepsilon \rightarrow 0} \iint_{F \times F} \frac{1}{2 \varepsilon} \mathbb{I}_{\operatorname{Diag}_{\varepsilon}}(\lambda, \tau) e^{2 \pi \cdot 2 a(\lambda+\tau)} d \rho(\lambda) d \rho(\tau) \\
& =c_{\delta} \liminf _{\varepsilon \rightarrow 0} \int_{F} \frac{1}{2 \varepsilon} \rho_{2 a}((\tau-\varepsilon, \tau+\varepsilon) \cap F) d \rho_{2 a}(\tau) \\
& =c_{\delta} \liminf _{\varepsilon \rightarrow 0} \int_{\mathbb{R}} \frac{1}{2 \varepsilon} \mu(\tau-\varepsilon, \tau+\varepsilon) d \mu(\tau),
\end{aligned}
$$


where $\mu$ is the restriction of $\rho_{2 a}$ to $F$, i.e. $\mu(\varphi)=\rho_{2 a}\left(\mathbb{I}_{F} \cdot \varphi\right)$ for any test-function $\varphi$. Notice that by the choice of $F, \mu(\mathbb{R})=\rho_{2 a}(F)>0$. By Lemma 4.1, the RHS of (23) is strictly positive, and we conclude that $L_{1}>0$.

4.3. Linear Variance. Consider again the first line of (17). Recall that, as we saw in Section 3.6. each term of the series in the RHS of (17) is non-negative. Therefore, by the monotone convergence theorem:

$$
\lim _{T \rightarrow \infty} \frac{V_{f}^{a, b}(T)}{2 T}=\frac{1}{8 \pi^{2}} \sum_{k \geq 1} \frac{1}{k^{2}} \lim _{T \rightarrow \infty} \int_{-2 T}^{2 T}\left(1-\frac{|x|}{2 T}\right)\left(S_{k}^{a, a}(x)-2 S_{k}^{a, b}(x)+S_{k}^{b, b}(x)\right) d x,
$$

provided that the limit of each term on the RHS exists. These limits can be computed using Observation 4.1 ;

$$
\lim _{T \rightarrow \infty} \frac{V_{f}^{a, b}(T)}{2 T}=\frac{1}{8 \pi^{2}} \sum_{k \geq 1} \frac{1}{k^{2}} \int_{\mathbb{R}}\left(S_{k}^{a, a}(x)-2 S_{k}^{a, b}(x)+S_{k}^{b, b}(x)\right) d x,
$$

which is finite by Lemma 4.2 ,

Lastly, we explain how to obtain the form of $L_{1}$ appearing in Remark 1.3. By monotone convergence theorem, we may take term-by-term limit as $T \rightarrow \infty$ in Proposition 2, and get:

$$
\lim _{T \rightarrow \infty} \frac{V_{f}^{a, b}(T)}{2 T}=\frac{1}{8 \pi^{3}} \sum_{k \geq 1} \frac{1}{k^{2}} \int_{\mathbb{R}}\left(p^{* k}(\lambda)\right)^{2} h_{k}^{a, b}(2 \lambda) d \lambda \in(0, \infty) .
$$

\section{Theorem 3; Super-Linear Variance}

In this section we prove the two items of Theorem 3, in reverse order.

5.1. Item (ii); Super-linear variance for particular $a, b$. Assume condition (3) holds for the particular $a$ and $b$ at hand. Fix a parameter $\delta>0$, and let $F=F_{\delta}$ be the set provided by Claim 7 . The premise ensures that, if $\delta$ is small enough, at least one of the measures $\left.(1+\lambda) \rho_{2 a}\right|_{F_{\delta}}$ and $\left.(1+\lambda) \rho_{2 b}\right|_{F_{\delta}}$ does not have $L^{2}$-density. WLOG assume it is the former. At first, assume also $\left.\rho_{2 a}\right|_{F}$ is not in $L^{2}$. Repeating the arguments of the Subsection 4.2 we get the lower bound

$$
\liminf _{T \rightarrow \infty} \frac{V_{f}^{a, b}(T)}{2 T} \geq c_{\delta} \int_{\mathbb{R}}|\mathcal{F}[\mu]|^{2}(x) d x,
$$

where $\mu=\left.\rho_{2 a}\right|_{F}$ and $c_{\delta}>0$. The LHS is therefore infinite, and so $L_{1}=\infty$.

We are left with the case that $\left.\lambda \rho_{2 a}\right|_{F_{\delta}}$ does not have $L^{2}$-density, but $\left.\rho_{2 a}\right|_{F_{\delta}}$ does (denote it by $\left.p_{2 a}\right)$. The argument is similar. Continuing from (22) and employing Claim 7 , we get

$$
\begin{aligned}
\liminf _{T \rightarrow \infty} \frac{V_{f}^{a, b}(T)}{2 T} & \geq c_{\delta} \liminf _{\varepsilon \rightarrow 0} \int_{F} \int_{F} \frac{1}{2 \varepsilon} \mathbb{I}_{(\tau-\varepsilon, \tau+\varepsilon)}(\lambda)(\lambda+\tau)^{2} p_{2 a}(\lambda) p_{2 a}(\tau) d \lambda d \tau \\
& \geq c_{\delta} \cdot 4 \int_{K} \lambda^{2} p_{2 a}(\lambda)^{2} d \lambda,
\end{aligned}
$$

where $K \subset F$ is compact. But, by our assumption, by choosing $K$ properly the last bound can be made arbitrarily large, so that $\lim _{T \rightarrow \infty} \frac{V_{f}^{a, b}(T)}{2 T}=\infty$. 
5.2. Item (i); Super-linear variance for almost all $a, b$. Let $\rho$ be such that the condition in item (i) holds. If $\rho$ has a singular component, then the condition in item (ii) holds for all possible $a, b$ and so $L_{1}(a, b)=\infty$ with no exceptions. Otherwise, $\rho$ has density $p(\lambda)$. Define the set

$$
E=\{(a, b): a, b \in J, a<b \text {, the condition in item (ii) fails for } a, b\} .
$$

If $E=\emptyset$, once again $L_{1}(a, b)=\infty$ for all $a, b \in J$ with no exceptions.

Assume then there is some $\left(a_{0}, b_{0}\right) \in E$. This means there exists $\lambda_{1}, \lambda_{2}$ such that for any pair of intervals $I_{1}, I_{2}$ such that $\lambda_{j} \in I_{j}(j=1,2)$, both the functions $\left(1+\lambda^{2}\right) e^{2 \pi \cdot 2 a_{0} \lambda} p(\lambda)$ and $\left(1+\lambda^{2}\right) e^{2 \pi \cdot 2 b_{0} \lambda} p(\lambda)$ are in $L^{2}\left(\mathbb{R} \backslash\left(I_{1} \cup I_{2}\right)\right)$, but at least one of them (WLOG, the former) is not in $L^{2}(\mathbb{R})$. Observe that the existence of such $\lambda_{1}, \lambda_{2}$ depends solely on $p(\lambda)$, and may therefore be regarded as independent of the point $\left(a_{0}, b_{0}\right) \in E$. Moreover, at least one among $\lambda_{1}$ and $\lambda_{2}$ (say, $\lambda_{1}$ ) is such that for any neighborhood $I$ containing it, $p \notin L^{2}(I)$.

Suppose now $a, b \in E$ are such that

$$
h_{1}^{a, b}\left(\lambda_{1}\right)>0,
$$

where $h_{1}^{a, b}(\lambda)=\left(l_{1}^{a}(\lambda) e^{2 \pi a \lambda}-l_{1}^{b}(\lambda) e^{2 \pi b \lambda}\right)^{2}$ is the function appearing in the the first term of our asymptotic formula, and in the lower bound in inequality (22). Recall $h_{1}^{a, b}$ is non-negative and has only two zeroes by Claim 5 .

We may choose $\delta>0$ smaller than the minimal distance between $\lambda_{1}$ and a zero of $h_{1}^{a, b}$, and then construct $F=F_{\delta}$ as in Claim 7. Certainly $\lambda_{1} \in F_{\delta}$, and so the measure $\mu=\left.\rho_{2 a}\right|_{F_{\delta}}$ is not in $L^{2}(\mathbb{R})$ (it is even not in $L^{2}(I)$ for any neighborhood $I$ of $\lambda_{1}$ ). Just as in subsection 4.2 we shall get

$$
\liminf _{T \rightarrow \infty} \frac{V_{f}^{a, b}(T)}{2 T} \geq c_{\delta} \int_{\mathbb{R}}|\mathcal{F}[\mu]|^{2}(x) d x=\infty .
$$

We end by showing that for a given point $\lambda_{1} \in \mathbb{R}$ and a given $a \in J$, the set of $b \in J$ which do not obey (24) is finite. Indeed, this is the set

$$
\left\{b \in J: h^{a, b}\left(\lambda_{1}\right)=0\right\}=\{b \in J: \varphi(a)=\varphi(b)\}
$$

where

$$
\varphi(y)=e^{2 \pi y \lambda_{1}} l_{1}^{y}\left(\lambda_{1}\right)=\frac{\partial}{\partial y}\left(\frac{e^{2 \pi \lambda_{1} y}}{r(2 i y)}\right) .
$$

Suppose the desired set is not finite. Since $\varphi$ is real-analytic, it must be constant on $J$. But then $r(2 i y)=\frac{e^{2 \pi \lambda_{1} y}}{c y+d}$ for some $c, d \in \mathbb{R}$, and the corresponding spectral density would satisfy condition (2) for all relevant $a, b$. This contradiction ends the proof.

\section{Appendix A. Uniform EXPONENTIAL DECAY OF TAILS}

In this appendix we prove Proposition 1, We follow closely [7, Proposition 5.1] and [10, Ch. 7] which prove similar concentration bounds, known as "Offord-type estimates". We rely on the following lemma, which follows either from [7, Lemma 6.1] or [10, Lemma 7.1.2]. 
Lemma A.1. If $\eta \sim \mathcal{N}_{\mathbb{C}}\left(0, \sigma^{2}\right)$, and $E$ is an event in the probability space with $\mathbb{P}(E)=p$, then:

$$
\left|\mathbb{E}\left(\chi_{E} \log |\eta|\right)\right| \leq p\left[\frac{p}{2}-2 \log p+\log \sigma\right] .
$$

Proof of Proposition 1. Take $\phi(z)=\phi_{T}(z)$ to be a real $C^{2}$ function, whose support is $\left[-\frac{1}{2}-T, T+\frac{1}{2}\right] \times\left[a^{\prime}, b^{\prime}\right]$ with $-\Delta<a^{\prime}<a<b<b^{\prime}<\Delta$, and which takes the value 1 on $R_{T}=[-T, T] \times[a, b)$. We may build such $\phi_{T}(z)$ that will obey also the bound $\|\Delta \phi\|_{L^{1}}<$ $10(T+b-a)$. Fix $s>0$. We are interested in dominating the probability of the event $A_{T}=\left\{n_{f}\left(R_{T}\right)>s T\right\}$. Write $p=p_{T}=\mathbb{P}\left(A_{T}\right)$.

We have

$$
n_{f}\left(R_{T}\right)<\frac{1}{2 \pi} \int \Delta \phi_{T}(z) \log |f(z)| d m(z)
$$

and therefore,

$$
\begin{aligned}
s T \cdot p & \leq \mathbb{E}\left(\chi_{A_{T}} n_{f}\left(R_{T}\right)\right) \leq \mathbb{E}\left(\chi_{A_{T}} \frac{1}{2 \pi} \int \Delta \phi(z) \log |f(z)| d m(z)\right) \\
& =\frac{1}{2 \pi} \int \Delta \phi \mathbb{E}\left(\chi_{A_{T}} \log |f(z)|\right) d m(z) \\
& \leq \frac{1}{2 \pi}\|\Delta \phi\|_{L^{1}} \sup _{z \in \mathbb{R} \times\left[a^{\prime}, b^{\prime}\right]} \mathbb{E}\left(\chi_{A_{T}} \log |f(z)|\right)
\end{aligned}
$$

The exchange of expectation and integral is justified by Fubini's theorem, as follows:

$$
\int_{D} \mathbb{E}|\Delta \phi(z) \cdot \log | f(z)|| d m(z)=\int_{D}|\Delta \phi(z)| \mathbb{E}|\log | f(z)||<\sup \mathbb{E}|\log | f(z)|| \cdot\|\Delta \phi\|_{L^{1}}<\infty .
$$

Applying lemma A.1 with $\eta=f(z)$, we get:

$$
\sup _{z \in \mathbb{R} \times\left[a^{\prime}, b^{\prime}\right]} \mathbb{E}\left(\chi_{A_{T}} \log |f(z)|\right)<p(\alpha-2 \log p) .
$$

for some constant $\alpha>0$ (depending on $\sup _{y \in\left[a^{\prime}, b^{\prime}\right]} \mathbb{E}|f(i y)|^{2}$ ). Putting all this together, we get:

$$
s T \cdot p \leq \frac{5}{\pi}(T+b-a) p\left(c_{1}-2 \log p\right),
$$

which leads to the exponential bound we strived for:

$$
\exists c, C>0 \text { such that } p_{T}=\mathbb{P}\left(n_{f}\left(R_{T}\right)>T s\right) \leq C e^{-c s}, \forall T \geq 1 \text {. }
$$

\section{ApPENDix B. JUSTIFICATION FOR CHANGING OPERATIONS}

In this section we prove Lemmas 3.4 and 3.5 . 
B.1. Proof of Lemma 3.4. We begin by showing (A-I) Let $1<p<2$ be arbitrary, and let $q>2$ be such that $\frac{1}{p}+\frac{1}{q}=1$. Hölder's inequality implies:

$$
\begin{aligned}
\int_{-T}^{T} \mathbb{E}\left|\frac{f^{\prime}(t+i a)}{f(t+i a)}\right| & \leq \int_{-T}^{T} \mathbb{E}\left[\left|f^{\prime}(t+i a)\right|^{q}\right]^{1 / q} \mathbb{E}\left[|f(t+i a)|^{-p}\right]^{1 / p} d t \\
& \leq \mathbb{E}\left[\left|f^{\prime}(i a)\right|^{q}\right]^{1 / q} \mathbb{E}\left[|f(i a)|^{-p}\right]^{1 / p} \cdot T<\infty,
\end{aligned}
$$

where finiteness follows from $f^{\prime}(i a)$ and $f(i a)$ being complex Gaussian random variables, thus having finite moments of any order.

We now turn to prove (A-II). We use the notation $f \lesssim g$ to stand for the inequality $f \leq C \cdot g$, where $C>0$ is a constant (which may vary from line to line). Similarly, $f \approx g$ stands for $f=C \cdot g$ with some $C>0$.

As before, let $1<p<2$ and take $q>2$ to obey $\frac{1}{p}+\frac{1}{q}=1$. By Hölder inequality we have

$$
\begin{aligned}
& \int_{-T}^{T} \int_{-T}^{T} \mathbb{E}\left|\frac{f^{\prime}(t+i a) f^{\prime}(s+i b)}{f(t+i a) f(s+i b)}\right| d t d s \\
& \leq \int_{-T}^{T} \int_{-T}^{T}\left[\mathbb{E}\left|f^{\prime}(t+i a) f^{\prime}(s+i b)\right|^{q}\right]^{1 / q}\left[\mathbb{E}|f(t+i a) f(s+i b)|^{-p}\right]^{1 / p} d t d s \\
& \lesssim \int_{-T}^{T} \int_{-T}^{T} \mathbb{E}\left[|f(t+i a) f(s+i b)|^{-p}\right]^{1 / p} d t d s .
\end{aligned}
$$

The last inequality is an application of Cauchy-Schwarz inequality and stationarity, as follows:

$$
\begin{aligned}
& {\left[\mathbb{E}\left|f^{\prime}(t+i a) f^{\prime}(s+i b)\right|^{q}\right]^{\frac{1}{q}}} \\
& \leq\left(\sqrt{\mathbb{E}\left|f^{\prime}(t+i a)\right|^{2 q} \mathbb{E}\left|f^{\prime}(s+i b)\right|^{2 q}}\right)^{\frac{1}{q}}=\left(\mathbb{E}\left[\left|f^{\prime}(i a)\right|^{2 q}\right] \mathbb{E}\left[\left|f^{\prime}(i b)\right|^{2 q}\right]\right)^{\frac{1}{2 q}}<\infty,
\end{aligned}
$$

where again finiteness follows from $f^{\prime}(i a)$ and $f^{\prime}(i b)$ being Gaussian.

Let

$$
A=\left\{(t, s) \in[-T, T]^{2}:|r(t-s+i a+i b)|^{2} \leq \frac{2}{3} r(2 i a) r(2 i b) .\right\} .
$$

We split the last integral in (25) into two parts: on $A$ and on $A^{c}=[-T, T]^{2} \backslash A$. For the integral on $A$ we use the following lemma:

Lemma B.1. Suppose $\xi_{1}, \xi_{2}$ are independent $\mathcal{N}_{\mathbb{C}}(0,1)$ random variables, and let $Z_{1}=\alpha \xi_{1}$ and $Z_{2}=\beta \xi_{1}+\gamma \xi_{2}$ where $\alpha, \beta, \gamma \in \mathbb{C} \backslash\{0\}$. Let $1<p<2$. Then

$$
\mathbb{E}\left[\left|Z_{1} Z_{2}\right|^{-p}\right] \leq|\alpha \gamma|^{-p} \Gamma\left(1-\frac{p}{2}\right)^{2}
$$

We include the proof in Section B.3. We apply Lemma B.1 with $Z_{1}=f(t+i a)$ and $Z_{2}=f(s+i b)$, which yields the choice of parameters $\alpha, \beta, \gamma$ so that

$$
\alpha=\sqrt{r(2 i a)}, \quad \alpha \bar{\beta}=\overline{r(t-s+i a+i b)}, \quad|\beta|^{2}+|\gamma|^{2}=r(2 i b) .
$$


In particular,

$$
|\alpha \gamma|=\sqrt{r(2 i a) r(2 i b)-|r(t-s+i a+i b)|^{2}} .
$$

Using this in Lemma B.1, we have:

$$
\begin{aligned}
\iint_{A} & \left(\mathbb{E}|f(t+i a) f(s+i b)|^{-p}\right)^{1 / p} d t d s \\
& \lesssim \iint_{A}\left(r(2 i a) r(2 i b)-|r(t-s+i a+i b)|^{2}\right)^{-p / 2} d t d s
\end{aligned}
$$

which is bounded by the definition of $A$. In order to bound the integration on $A^{c}$, we use another lemma (which is also proved in Section B.3).

Lemma B.2. Suppose $\xi_{1}, \xi_{2}$ are independent $\mathcal{N}_{\mathbb{C}}(0,1)$ random variables, and let $Z_{1}=\alpha \xi_{1}$ and $Z_{2}=\beta \xi_{1}+\gamma \xi_{2}$ where $\alpha, \beta, \gamma \in \mathbb{C} \backslash\{0\}$. Suppose $M>0$ is such that $\left|\frac{\gamma}{\beta}\right|<M$, and let $1<p<2$. Then there exists a constant $c>0$, depending only on $M$ and $p$, such that

$$
\mathbb{E}\left[\frac{1}{\left|Z_{1} Z_{2}\right|^{p}}\right] \leq \frac{c}{|\alpha \beta|^{p}}\left|\frac{\gamma}{\beta}\right|^{2-2 p} .
$$

We apply this lemma again to $Z_{1}=f(t+i a)$ and $Z_{2}=f(s+i b)$, so the choice of parameters in (26) remains valid. Thus,

$$
\left|\frac{\gamma}{\beta}\right|^{2}=\frac{r(2 i a) r(2 i b)}{|r(t-s+i a+i b)|^{2}}-1
$$

is uniformly bounded for $(t, s) \in A^{c}$. Applying Lemma B.2 we get that for some $c>0$,

$$
\begin{aligned}
\iint_{A^{c}} & \left(\mathbb{E}|f(t+i a) f(s+i b)|^{-p}\right)^{1 / p} d t d s \\
& \lesssim \iint_{A^{c}}\left(\frac{c}{|r(t-s+i a+i b)|^{p}} \frac{\left(r(2 i a) r(2 i b)-|r(t-s+i a+i b)|^{2}\right)^{1-p}}{|r(t-s+i a+i b)|^{2-2 p}}\right)^{\frac{1}{p}} d t d s \\
& \lesssim \iint_{A^{c}}\left(r(2 i a) r(2 i b)-|r(t-s+i a+i b)|^{2}\right)^{-\frac{p-1}{p}} d t d s \\
& \lesssim \int_{\tilde{A}^{c}}\left(r(2 i a) r(2 i b)-|r(x+i a+i b)|^{2}\right)^{-\frac{p-1}{p}} d x,
\end{aligned}
$$

where $\tilde{A}^{c}$ is the one-dimensional set

$$
\tilde{A}^{c}=\left\{x \in[-T, T]:|r(x+i a+i b)|^{2}>\frac{2}{3} r(2 i a) r(2 i b)\right\} .
$$

The last inequality in (27) is obtained by a simple change of varibales. One step before that in (27) we bounded $|r(t-s+i a+i b)|^{-1}$ from above by a constant, using the definition of $A^{c}$. 
Before continuing, we notice that

$$
\begin{aligned}
|r(x+i a+i b)|^{2} & =\left|\int_{\mathbb{R}} e^{-2 \pi i x \lambda} e^{2 \pi \cdot(a+b) \lambda} d \rho(\lambda)\right|^{2} \\
& \leq\left(\int_{\mathbb{R}} e^{2 \pi \cdot(a+b) \lambda} d \rho(\lambda)\right)^{2}\left(=r(i a+i b)^{2}\right) \\
& \leq \int_{\mathbb{R}} e^{2 \pi \cdot 2 a \lambda} d \rho(\lambda) \cdot \int_{\mathbb{R}} e^{2 \pi \cdot 2 a \lambda} d \rho(\lambda) \\
& =r(2 i a) r(2 i b),
\end{aligned}
$$

and the inequality is sharp when $a \neq b$ (see also part 2 of Lemma 3.3 below). Therefore, if $a \neq b$, the last integral in (27) is finite.

In case $a=b$, there may be only a finite number of isolated points $x_{0}$ for which $\mid r\left(x_{0}+\right.$ $2 i a)\left.\right|^{2}=r(2 i a)^{2}$. Taylor expansion near any of those points gives $|r(x+2 i a)|^{2}=r(2 i a)^{2}-$ $C\left(x-x_{0}\right)^{2}+o\left(\left(x-x_{0}\right)^{2}\right)$ as $x$ tends to $x_{0}$ (here $C \geq 0$ since $|r(x+2 i a)|^{2} \leq r(2 i a)^{2}$ by taking $a=b$ in (28) $)$. So, in this case the finiteness of the integral (27) is equivalent to that of

$$
\int_{\left|x-x_{0}\right|<\delta}\left(x-x_{0}\right)^{-2(p-1) / p} d x
$$

(with some $\delta>0$ ), which is indeed finite for $1<p<2$.

B.2. Proof of Lemma 3.5. We will justify in detail the second item, as the proof of the first is similar and simpler.

Fix $(t, s) \in[-T, T]^{2}$. We may assume that $|r(t-s+i a+i b)|^{2}<r(2 i a) r(2 i b)$ as the set of $(t, s) \in[-T, T]^{2}$ where this inequality does not hold is of measure zero (see discussion following (28) $)$. Consider the random variables

$$
U\left(h_{1}, h_{2}\right)=\frac{\log \left|f\left(t+i a+i h_{1}\right)\right|-\log |f(t+i a)|}{h_{1}} \cdot \frac{\log \left|f\left(s+i b+i h_{2}\right)\right|-\log |f(s+i b)|}{h_{2}} .
$$

These are well-defined for $0<h_{1}<\delta_{1}$ and $0<h_{2}<\delta_{2}$, where $\delta_{1}, \delta_{2}$ are properly chosen numbers (we use here that almost surely, there are no zeroes on the vertical lines $\{t\} \times\left[a, a+\delta_{1}\right]$ and $\{s\} \times\left[b, b+\delta_{2}\right]$, as was explained in Section 3.2). Notice that almost surely,

$$
\lim _{h_{1} \rightarrow 0+h_{2} \rightarrow 0+} U\left(h_{1}, h_{2}\right)=\frac{\partial^{2}}{\partial a \partial b}[\log |f(t+i a)| \log |f(s+i b)|] .
$$

Our goal (B-II) can be understood as convergence in $L^{1}(\mathbb{P})$ of the above limit. This will follow if the family $\left\{U\left(h_{1}, h_{2}\right)\right\}_{h_{1}, h_{2}}$ is uniformly integrable, i.e., if for every $\varepsilon>0$ there exists $k>0$ such that for all relevant $h_{1}, h_{2}$ it holds that

$$
\mathbb{E}\left(\left|U\left(h_{1}, h_{2}\right)\right| \mathbb{I}_{\left\{\left|U\left(h_{1}, h_{2}\right)\right| \geq k\right\}}\right)<\varepsilon .
$$

Uniform integrability, in turn, would follow from the following statement: 2

\footnotetext{
${ }^{2}$ Indeed, suppose (29) holds. Denoting by $q$ the number such that $\frac{1}{p}+\frac{1}{q}=1$, we apply Hölder's inequality to get:

$$
\mathbb{E}\left(\left|U\left(h_{1}, h_{2}\right)\right| \mathbb{I}\left\{\left|U\left(h_{1}, h_{2}\right)\right| \geq k\right\}\right) \leq\left(\mathbb{E}\left|U\left(h_{1}, h_{2}\right)\right|^{p}\right)^{1 / p} \mathbb{P}\left(\left|U\left(h_{1}, h_{2}\right)\right| \geq k\right)^{1 / q} \lesssim \frac{1}{k^{p / q}},
$$
}




$$
\exists p>1: \sup _{h_{1}, h_{2}} \mathbb{E}\left|U\left(h_{1}, h_{2}\right)\right|^{p}<\infty .
$$

Applying the Newton-Leibniz formula, the bound (8) and Jensen's inequality we get:

$$
\begin{aligned}
\left|U\left(h_{1}, h_{2}\right)\right| & =\left|\frac{1}{h_{1} h_{2}} \int_{0}^{h_{2}} \int_{0}^{h_{1}} \frac{\partial}{\partial y_{1}} \log \right| f\left(t+i a+i y_{1}\right)\left|\frac{\partial}{\partial y_{2}} \log \right| f\left(s+i b+i y_{2}\right)\left|d y_{1} d y_{2}\right| \\
& \leq \int_{0}^{h_{2}} \int_{0}^{h_{1}}\left|\frac{f^{\prime}}{f}\left(t+i a+i y_{1}\right)\right|\left|\frac{f^{\prime}}{f}\left(s+i b+i y_{1}\right)\right| \frac{d y_{1}}{h_{1}} \frac{d y_{2}}{h_{2}} \\
& \leq\left(\int_{0}^{h_{2}} \int_{0}^{h_{1}}\left|\frac{f^{\prime}}{f}\left(t+i a+i y_{1}\right)\right|^{p}\left|\frac{f^{\prime}}{f}\left(s+i b+i y_{1}\right)\right|^{p} \frac{d y_{1}}{h_{1}} \frac{d y_{2}}{h_{2}}\right)^{1 / p} .
\end{aligned}
$$

Taking $1<p^{\prime}<\frac{2}{p}$ and $q^{\prime}$ such that $\frac{1}{p^{\prime}}+\frac{1}{q^{\prime}}=1$, we apply Hölder's inqeuality to bound the last expression by

$$
\begin{aligned}
& \left(\int_{0}^{h_{2}} \int_{0}^{h_{1}} \mathbb{E}\left|f^{\prime}\left(t+i a+i y_{1}\right) f^{\prime}\left(s+i b+i y_{2}\right)\right|^{p q^{\prime}} \frac{d y_{1}}{h_{1}} \frac{d y_{2}}{h_{2}}\right)^{\frac{1}{p q^{\prime}}} \\
& \quad \times\left(\int_{0}^{h_{2}} \int_{0}^{h_{1}} \mathbb{E}\left|f\left(t+i a+i y_{1}\right) f\left(s+i b+i y_{2}\right)\right|^{-p p^{\prime}} \frac{d y_{1}}{h_{1}} \frac{d y_{2}}{h_{2}}\right)^{\frac{1}{p p^{\prime}}}
\end{aligned}
$$

Using Cauchy-Schwarz, the first integral is bounded by

$$
\left(\max _{y_{1} \in\left[0, \delta_{1}\right]} \mathbb{E}\left|f^{\prime}\left(t+i a+i y_{1}\right)\right|^{2 p q^{\prime}} \max _{y_{2} \in\left[0, \delta_{2}\right]} \mathbb{E}\left|f^{\prime}\left(s+i b+i y_{2}\right)\right|^{2 p q^{\prime}}\right)^{\frac{1}{2 p q^{\prime}}},
$$

which is finite and independent of $h_{1}$ and $h_{2}$. Applying Lemma B.1 with the same choice of parameters as before, we may bound the second integral (up to a constant factor depending on $p$ and $p^{\prime}$ ) by:

$$
\begin{aligned}
& \left(\int_{0}^{h_{1}} \int_{0}^{h_{2}}\left\{r\left(2 i a+2 i y_{1}\right) r\left(2 i b+2 i y_{2}\right)-\left|r\left(t-s+i a+i b+i y_{2}+i y_{2}\right)\right|^{2}\right\}^{-\frac{p p^{\prime}}{2}} \frac{d y_{1}}{h_{1}} \frac{d y_{2}}{h_{2}}\right)^{\frac{1}{p p^{\prime}}} \\
& \lesssim \max _{y_{1} \in\left[0, \delta_{1}\right], y_{2} \in\left[0, \delta_{2}\right]}\left\{r\left(2 i a+2 i y_{1}\right) r\left(2 i b+2 i y_{2}\right)-\left|r\left(t-s+i a+i b+i y_{2}+i y_{2}\right)\right|^{2}\right\}^{-\frac{1}{2}},
\end{aligned}
$$

which is again finite and independent of $h_{1}$ and $h_{2}$. Our proof is complete.

B.3. Proofs of auxiliary Lemmas. This part is dedicated to prove Lemmas B.1 and B.2 that were used earlier in this section.

Proof of Lemma B.1. Using the notations in the statement of the Lemma, we have:

$$
\mathbb{E}\left[\left|Z_{1} Z_{2}\right|^{-p}\right]=\frac{1}{\pi^{2}} \iint_{\mathbb{C}^{2}}\left|\alpha \xi_{1}\left(\beta \xi_{1}+\gamma \xi_{2}\right)\right|^{-p} e^{-\left|\xi_{1}\right|^{2}-\left|\xi_{2}\right|^{2}} d m\left(\xi_{1}\right) d m\left(\xi_{2}\right)
$$

\footnotetext{
so the definition of uniform integrability is satisfied.
} 
Now, by the Hardy-Littlewood re-arrangement inequality, we have:

$$
\begin{aligned}
& \frac{1}{\pi} \int_{\mathbb{C}}\left|\beta \xi_{1}+\gamma \xi_{2}\right|^{-p} e^{-\left|\xi_{2}\right|^{2}} d m\left(\xi_{2}\right) \leq|\gamma|^{-p} \cdot \frac{1}{\pi} \int_{\mathbb{C}}\left|\xi_{2}\right|^{-p} e^{-\left|\xi_{2}\right|^{2}} d m\left(\xi_{2}\right) \\
& \quad=|\gamma|^{-p} \Gamma\left(1-\frac{p}{2}\right) .
\end{aligned}
$$

So,

$$
\begin{aligned}
\mathbb{E}\left[\left|Z_{1} Z_{2}\right|^{-p}\right] & \leq|\alpha \gamma|^{-p} \Gamma\left(1-\frac{p}{2}\right) \cdot \frac{1}{\pi} \int_{\mathbb{C}}\left|\xi_{1}\right|^{-p} e^{-\left|\xi_{1}\right|^{2}} d m\left(\xi_{1}\right) \\
& =|\alpha \gamma|^{-p} \Gamma\left(1-\frac{p}{2}\right)^{2} .
\end{aligned}
$$

Proof of Lemma B.2. In this proof, the constant hidden by the "§" and " $\sim$ " notation depends only on $M$ and $p$.

We begin by writing-out the desired expectation explicitly.

$$
\begin{aligned}
\mathbb{E}\left[\left|Z_{1} Z_{2}\right|^{-p}\right] & =|\alpha \beta|^{-p} \mathbb{E}\left[\left|\xi_{1}^{2}+\frac{\gamma}{\beta} \xi_{1} \xi_{2}\right|^{-p}\right] \\
& =|\alpha \beta|^{-p} \cdot \frac{1}{\pi^{2}} \iint_{\mathbb{C}^{2}}\left|z^{2}+\frac{\gamma}{\beta} z w\right|^{-p} e^{-|z|^{2}-|w|^{2}} d m(z) d m(w) \\
& =|\alpha \beta|^{-p} \pi^{-2} \int_{\mathbb{C}}|z|^{-p}\left(\int_{\mathbb{C}}\left|z+\frac{\gamma}{\beta} w\right|^{-p} e^{-|w|^{2}} d m(w)\right) e^{-|z|^{2}} d m(z) .
\end{aligned}
$$

We bound the inner integral as follows:

$$
\begin{aligned}
& \int_{\mathbb{C}}\left|z+\frac{\gamma}{\beta} w\right|^{-p} e^{-|w|^{2}} d m(w) \\
& \lesssim \int_{|w| \leq \frac{1}{2}\left|\frac{\beta}{\gamma} z\right|}|z|^{-p} e^{-|w|^{2}} d m(w)+|z|^{-p} e^{-\frac{1}{4}\left|\frac{\beta}{\gamma} z\right|^{2}}\left|\frac{\beta}{\gamma} z\right|^{2}+\int_{|w|>2\left|\frac{\beta}{\gamma} z\right|}\left|\frac{\gamma}{\beta} w\right|^{-p} e^{-|w|^{2}} d m(w) \\
& \bar{\sim}|z|^{-p}\left(1-e^{-\frac{1}{4}\left|\frac{\beta}{\gamma} z\right|^{2}}\right)+\left|\frac{\beta}{\gamma}\right|^{2}|z|^{2-p} e^{-\frac{1}{4}\left|\frac{\beta}{\gamma} z\right|^{2}}+\left|\frac{\beta}{\gamma}\right|^{p} I\left(\left|\frac{\beta}{\gamma} z\right|\right),
\end{aligned}
$$

where

$$
I(s)=\int_{|w|>2 s}|w|^{-p} e^{-|w|^{2}} d m(w) \lesssim \begin{cases}1, & 0<s \leq 1 \\ s^{-p} e^{-4 s^{2}}, & s>1 .\end{cases}
$$

The last bound is achieved by changing to polar coordinates, as follows:

$$
I(s) \approx \int_{2 s}^{\infty} r^{-p+1} e^{-r^{2}} d r \lesssim s^{-p+1} \int_{2 s}^{\infty} e^{-r^{2}} d r \leq s^{-p+1} \frac{1}{2 s} e^{-4 s^{2}}
$$


Returning to the double integral in (30), we have:

$$
\begin{aligned}
& \mathbb{E}\left[\left|\xi_{1}^{2}+\frac{\gamma}{\beta} \xi_{1} \xi_{2}\right|^{-p}\right] \\
& \lesssim \int_{\mathbb{C}}\left\{|z|^{-2 p}\left(1-e^{-\frac{1}{4}\left|\frac{\beta}{\gamma} z\right|^{2}}\right)+\left|\frac{\beta}{\gamma}\right|^{2}|z|^{2-2 p} e^{-\frac{1}{4}\left|\frac{\beta}{\gamma} z\right|^{2}}+|z|^{-p}\left|\frac{\beta}{\gamma}\right|^{p} I\left(\left|\frac{\beta}{\gamma} z\right|\right)\right\} e^{-|z|^{2}} d m(z)
\end{aligned}
$$

This is the sum of three integrals, which we bound separately. For the first, we have:

$$
\begin{aligned}
\int_{\mathbb{C}}|z|^{-2 p}\left(1-e^{-\frac{1}{4}\left|\frac{\beta}{\gamma} z\right|^{2}}\right) e^{-|z|^{2}} d m(z) \\
\lesssim \int_{|z| \leq\left|\frac{\gamma}{\beta}\right|}\left|\frac{\beta}{\gamma}\right|^{2}|z|^{2-2 p} d m(z)+\int_{|z|>\left|\frac{\gamma}{\beta}\right|^{2}}|z|^{-2 p} e^{-|z|^{2}} d m(z) \\
\quad \approx\left|\frac{\beta}{\gamma}\right|^{2}\left|\frac{\gamma}{\beta}\right|^{4-2 p}+O(1) \approx\left|\frac{\gamma}{\beta}\right|^{2-2 p}
\end{aligned}
$$

Denote $A=1+\frac{1}{4}\left|\frac{\beta}{\gamma}\right|^{2}$. Before estimating the second integral, we compute

$$
\begin{array}{rlr}
\int_{\mathbb{C}}|z|^{2-2 p} e^{-A|z|^{2}} d m(z) & \\
& \approx \int_{0}^{\infty} r^{2-2 p} e^{-A r^{2}} r d r & {[r=|z|]} \\
& =\frac{1}{2 A} \int_{0}^{\infty}\left(\frac{s}{A}\right)^{1-p} e^{-s} d s & {\left[s=A r^{2}\right]} \\
& \approx A^{-(2-p)} &
\end{array}
$$

Thus, the second integral is

$$
\left|\frac{\beta}{\gamma}\right|^{2} \int_{\mathbb{C}}|z|^{2-2 p} e^{-\left(1+\frac{1}{4}\left|\frac{\beta}{\gamma}\right|^{2}\right)|z|^{2}} d m(z) \approx\left|\frac{\beta}{\gamma}\right|^{2}\left(1+\frac{1}{4}\left|\frac{\beta}{\gamma}\right|^{2}\right)^{-(2-p)} \bar{\sim}\left|\frac{\gamma}{\beta}\right|^{2-2 p} .
$$

For the third integral we first compute

$$
\begin{array}{rlr}
\int_{|z|>\left|\frac{\gamma}{\beta}\right|}|z|^{-2 p} e^{-A|z|^{2}} d m(z) & \\
& \approx \int_{\left|\frac{\gamma}{\beta}\right|}^{\infty} r^{-2 p} e^{-A r^{2}} r d r & {[r=|z|]} \\
& =\frac{1}{2 A} \int_{A\left|\frac{\gamma}{\beta}\right|}^{\infty}\left(\frac{s}{A}\right)^{-p} e^{-s} d s & \\
& \left.\lesssim A^{p-1} \int_{1 / 4}^{\infty} s^{-p} e^{-s} d s \approx A^{2}\right]
\end{array}
$$


Finally, the third integral is

$$
\begin{aligned}
& \left|\frac{\beta}{\gamma}\right|^{p} \int_{\mathbb{C}}|z|^{-p} I\left(\left|\frac{\beta}{\gamma} z\right|\right) e^{-|z|^{2}} d m(z) \\
& \quad \approx\left|\frac{\beta}{\gamma}\right|^{p}\left\{\int_{|z|<\left|\frac{\gamma}{\beta}\right|}|z|^{-p} e^{-|z|^{2}} d m(z)+\left|\frac{\beta}{\gamma}\right|^{-p} \int_{|z|>\left|\frac{\gamma}{\beta}\right|}|z|^{-2 p} e^{-\left(1+4\left|\frac{\beta}{\gamma}\right|^{2}\right)|z|^{2}} d m(z)\right\} \\
& \quad \approx\left|\frac{\beta}{\gamma}\right|^{p}\left|\frac{\gamma}{\beta}\right|^{2-p}+\left(1+4\left|\frac{\beta}{\gamma}\right|^{2}\right)^{p-1} \approx\left|\frac{\gamma}{\beta}\right|^{2-2 p} .
\end{aligned}
$$

The proof is complete.

\section{Appendix C. Moments of the CharaCteristic FunCtion}

Here we prove Lemma 4.3, which estimates moments of the characteristic function (or Fourier transform) of a probability distribution. We adapt the proof of the Central Limit Theorem appearing in [8, Ch. XV.5].

Proof of Lemma 4.3. Write $G(x)=\mathcal{F}[g](x)$. We may assume that $\int_{\mathbb{R}} \lambda g(\lambda)=0$ (otherwise we shall consider, instead of $g$, the function $g_{\mu}(\lambda)=g(\lambda+\mu)$ where $\mu:=\int_{\mathbb{R}} \lambda g(\lambda) d \lambda$. There is no penalty since $\left|\mathcal{F}\left[g_{\mu}\right](x)\right|=|\mathcal{F}[g](x)|$ for all $\left.x \in \mathbb{R}\right)$. By assumption $($ a), $G(x)$ is thrice differentiable, and by the above assumptions $G(0)=1$ and $G^{\prime}(0)=0$.

To prove the lemma, it is enough to show that

$$
\lim _{m \rightarrow \infty} \sqrt{m} \int_{\mathbb{R}}|G(x)|^{m} d x \text { exists and is finite. }
$$

Notice that $\sqrt{m} \int_{\mathbb{R}}|G(x)|^{m} d x=\int_{\mathbb{R}}|G(x / \sqrt{m})|^{m} d x$, and so it is enough to show that

$$
\left.\lim _{m \rightarrow \infty} \int_{\mathbb{R}}|| G\left(\frac{x}{\sqrt{m}}\right)\right|^{m}-e^{-\frac{\alpha x^{2}}{2}} \mid d x=0,
$$

for some value of $\alpha>0$, which in fact is $\alpha:=G^{\prime \prime}(0)$.

We shall achieve (31) by splitting the integral into three parts, and showing each could be made less than a given $\varepsilon>0$ if $m \geq \nu$ is chosen large enough.

Fix $R>0$ (to be determined later). By Taylor expansion,

$$
G(x)=G(0)+x G^{\prime}(0)+\frac{x^{2}}{2} G^{\prime \prime}(0)+o\left(x^{2}\right)=1+\frac{\alpha x^{2}}{2}+o\left(x^{2}\right), x \rightarrow 0
$$

and so $|G(x / \sqrt{m})|^{m} \rightarrow e^{-\alpha x^{2} / 2}$ as $m \rightarrow \infty$, uniformly in $x \in[-R, R]$. Thus the integral in (31) computed on $[-R, R]$ converges to zero as $m \rightarrow \infty$.

From the expansion (32) we get

$$
\exists \delta>0 \forall|x|<\delta:|G(x)| \leq e^{-\frac{\alpha x^{2}}{4}} .
$$

Consider the integration in (31) for $R \leq|x| \leq \delta \sqrt{m}$. For such $x$ we have $|G(x / \sqrt{m})|^{m} \leq$ $e^{-\frac{\alpha x^{2}}{4}}$, and so the integrand is less than $2 e^{-\frac{\alpha x^{2}}{4}}$. Choosing $R$ so that $4 \int_{R}^{\infty} e^{-\frac{\alpha x^{2}}{4}}<\varepsilon$ will satisfy our needs. 
Lastly, consider the integration on $\delta \sqrt{m} \leq|x|<\infty$. By properties of Fourier transform, $\eta:=\sup _{|x| \geq \delta}|G(x)| \in(0,1)$. Thus

$$
\left.\int_{|x| \geq \delta \sqrt{m}}|| G\left(\frac{x}{\sqrt{m}}\right)\right|^{m}-\left.e^{-\frac{\alpha x^{2}}{2}}\left|d x \leq \eta^{m-\nu} \sqrt{m} \int_{\mathbb{R}}\right| G\right|^{\nu}+\int_{|x| \geq \delta \sqrt{m}} e^{-\frac{\alpha x^{2}}{2}} d x<\varepsilon,
$$

for $m$ large enough. Here we have used condition (b),

\section{REFERENCES}

[1] J.-M. Azaïs, J.R. León, CLT for crossings of random trigonometric polynomials, Electron. J. Probab. 18 (2013), no. 68, 1-17. ISSN: 1083-6489.

[2] J. Buckley and N. Feldheim, Variance and CLT for the winding of a planar stationary Gaussian process, in final preparation.

[3] D. Chambers and E. Slud, Central limit theorems for nonlinear functionals of stationary Gaussian processes, Probab. Th. Rel. Fields 80 (1989) 323-346.

[4] H. Cramér and M.R. Leadbetter, Stationary and Related Stochastic Processes, Wiley series in Probability and Mathematical Statistics, 1967.

[5] J. Cuzick, A central limit theorem for the number of zeros of a stationary Gaussian process, Ann. Probab. 4 (1976) 547-556.

[6] R. Durrett, Probability: Theory and Examples, Cambridge University Press, fourth edition (2010).

[7] N. Feldheim, Zeroes of Gaussian Analytic Functions with Translation-Invariant Distribution, Israel Journal of Mathematics, 2012.

[8] W. Feller, An Introduction to Probability Theory and Its Applications, Vol. 2, 2nd edition, John Wiley and Sons, 1971.

[9] A. Granville, I. Wigman, The distribution of the zeros of random trigonometric polynomials, American Journal of Mathematics Vol. 133 (2), 2011, pp. 295-357.

[10] J.B. Hough, M. Krishnapur, Y. Peres and B. Virag, Zeroes of Gaussian Analytic Functions and Determinantal Processes, University Lecture Series, 51. American Mathematical Society, Providence, RI, 2009.

[11] M. Kac, On the average number fo real roots of a random algebraic equation, Bull. Amer. Math. Soc. 18 (1943), 29-35.

[12] Y. Katznelson, An Introduction to Harmonic Analysis (Third Edition), Cambridge University Press, 2004.

[13] M.F. Kratz, Level crossings and other level functionals of stationary Gaussian processes, Probability Surveys, 3 (2006), 230-288.

[14] F. Nazarov and M. Sodin, Fluctuations in Random Complex Zeroes: Asymptotic Normality Revisited, International Math. Research Notices Advance Access (2011).

[15] R.E.A.C. Paley and N. Wiener, Fourier Transforms in the Complex Domain, American Mathematical Sociey Colloquium Publications, vol. XIX (1967) 163-178.

[16] V.I. Piterbarg, Asymptotic Methods in the Theory of Gaussian Processses and Fields, Translations of Mathematical Monographs Vol. 148, American Mathematical Society, Providence, RI, 1996.

[17] S.O. Rice, Mathematical analysis of random noise, Bell Sys. Tech. Jour. 24 (1945), 46-156.

[18] E. Slud, Multiple Wiener-Ito integral expansions for level-crossing-count functionals, Prob. Th. Rel. Fields 87 (1991) 349-364.

[19] M. Sodin and B. Tsirelson, Random complex zeroes. I. Asymptotic normality. Israel J. Math. 144 (2004), 125-149. 